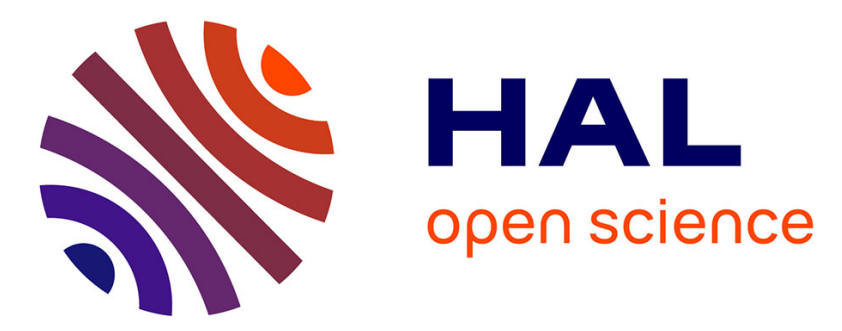

\title{
Acceleration in Convex Data-Flow Analysis
}

Jérôme Leroux, Grégoire Sutre

\section{- To cite this version:}

Jérôme Leroux, Grégoire Sutre. Acceleration in Convex Data-Flow Analysis. FSTTCS, 2007, New Delhi, India. pp.520-531, 10.1007/978-3-540-77050-3_43 . hal-00346295

\section{HAL Id: hal-00346295 \\ https://hal.science/hal-00346295}

Submitted on 15 Dec 2008

HAL is a multi-disciplinary open access archive for the deposit and dissemination of scientific research documents, whether they are published or not. The documents may come from teaching and research institutions in France or abroad, or from public or private research centers.
L'archive ouverte pluridisciplinaire HAL, est destinée au dépôt et à la diffusion de documents scientifiques de niveau recherche, publiés ou non, émanant des établissements d'enseignement et de recherche français ou étrangers, des laboratoires publics ou privés. 


\title{
Acceleration in Convex Data-Flow Analysis
}

\author{
Jérôme Leroux and Grégoire Sutre \\ LaBRI, Université de Bordeaux, CNRS \\ Domaine Universitaire, 351, cours de la Libération, 33405 Talence, France \\ \{leroux, sutre\}@labri.fr
}

\begin{abstract}
In abstract interpretation-based data-flow analysis, widening operators are usually used in order to speed up the iterative computation of the minimum fix-point solution (MFP). However, the use of widenings may lead to loss of precision in the analysis. Acceleration is an alternative to widening that has mainly been developed for symbolic verification of infinite-state systems. Intuitively, acceleration consists in computing the exact effect of some controlflow cycle in order to speed up reachability analysis. This paper investigates acceleration in convex data-flow analysis of systems with real-valued variables where guards are convex polyhedra and assignments are translations. In particular, we present a simple and algorithmically efficient characterization of MFPacceleration for cycles with a unique initial location. We also show that the MFPsolution is a computable algebraic polyhedron for systems with two variables.
\end{abstract}

\section{Introduction}

Formal verification of safety properties on a system is usually based on the automatic (or manual) generation of invariants of the system. Invariants are over-approximations of the set of all reachable configurations in the system. This over-approximation must be precise enough in order to determine which safety properties are satisfied by the system. Data-flow analysis, and in particular abstract interpretation [CC77], provides a powerful framework to develop analysis for computing such invariants.

For systems with numerical variables, linear relation analysis aims at computing invariants expressing linear relationships between variables [Kar76, CH78, Min01, SSM04, BHRZ05]. The desired invariant corresponds to the minimum fix-point (MFP) solution of the system's approximate semantics in some numerical domain, and it may be computed by Kleene fix-point iteration. However, the computation may diverge and widening/narrowing operators [CC77, CC92] are often used in order to enforce convergence at the expense of precision. This may lead to invariants that are too coarse to prove the desired safety properties on the system to be verified.

Acceleration is an alternative to widening that has mainly been developed for symbolic verification of infinite-state systems [BW94, CJ98, FIS03, FL02, BIL06]. Intuitively, acceleration consists in computing the exact effect of some control-flow cycle in order to speed up Kleene fix-point computations in reachability analysis. Accelerated symbolic model checkers such as LASH, TREX, and FAST successfully implement this approach. While being more precise than widening, acceleration is also more computationally expensive. 
Our contribution. We aim at developing methods that speed up the iterative computation of the MFP-solution, without any loss of precision. We focus on a class of systems with real-valued variables, the so-called guarded translation systems (GTSs). This class intuitively represents programs where conditions are closed convex sets and transformations are restricted to translations. We investigate acceleration of data-flow analysis for this class in the complete lattice of closed convex subsets of $\mathbb{R}^{n}$. To discuss computability issues, we devote particular attention to the class of rational polyhedral GTSs, where conditions are rational polyhedra and translation vectors are rational.

Recast in our setting, the (exact) acceleration techniques mentioned above consist in computing the merge over all path (MOP) solution along some (simple) cycle, which we call MOP-acceleration. We show that the MOP-acceleration of any cycle is an effectively computable rational polyhedron for rational polyhedral GTSs. However MOPacceleration is not in general sufficient to guarantee termination of the Kleene fix-point iteration, even for cyclic GTSs. We therefore investigate MFP-acceleration, which basically amounts to computing the MFP-solution of the system restricted to a given cycle. In other words, MFP-acceleration directly gives the MFP-solution for cyclic GTSs.

We obtain a surprisingly simple expression of the MFP-acceleration for cycles with a unique initial location. For rational polyhedral GTSs, this characterization shows that the MFP-acceleration is an effectively computable rational polyhedron for these cycles. This result cannot be extended to arbitrary cycles, as we give a 3-dim (i.e. three realvalued variables) cyclic example where the MFP-solution is not a polyhedron. We then focus on 2-dim GTSs and we prove that the MFP-solution is an effectively computable algebraic polyhedron (i.e. with algebraic coefficients) for general rational polyhedral 2-dim GTSs. Even for cyclic GTSs in this class, the polyhedral MFP-solution can be non-rational.

Related work. Karr introduced in [Kar76] an algorithm for computing the exact MFPsolution in the lattice of linear equalities. In [CH78], Cousot and Halbwachs framed linear relation analysis as an abstract interpretation and provided the first widening operator over the lattice of rational polyhedra. This approach only provides an overapproximation of the MFP-solution. Many refinements of this original widening operator have since been studied [BHRZ05] to limit the loss of precision. Recently Gonnord and Halbwachs [GH06] introduced the notion of abstract-acceleration as a complement to widening for linear relation analysis. We show that while maintaining the same computational complexity, our MFP-acceleration is "better" than abstract-acceleration in the sense that MFP-acceleration enforces convergence of the Kleene fix-point iteration strictly more often than abstract-acceleration. On another hand [GH06] also investigates acceleration of multiple loops and the combination of translations and resets.

Outline. The rest of the paper is organized as follows. Section 2 recalls some background material on lattices and convex sets. We introduce guarded translation systems in section 3, along with MOP-acceleration and MFP-acceleration for these systems. We present in sections 4 and 5 our results on MOP-acceleration and MFP-acceleration for guarded translation systems. Section 6 is devoted to the MFP-solution of general guarded translation systems in dimension not greater than 2. Most proofs are only sketched in the paper, but detailed proofs are given in appendix. This paper is the long version of our FSTTCS 2007 paper. 


\section{The Complete Lattice of Closed Convex Sets}

\subsection{Numbers, lattices and languages}

The paper follows the ISO 31-11 international standard for mathematical notation. We respectively denote by $\mathbb{Z}, \mathbb{Q}$ and $\mathbb{R}$ the usual sets of integers, rationals and real numbers. Recall that a (real) algebraic number is any real number that is the root of some nonzero polynomial with rational coefficients. We write $\mathbb{A}$ the set of all (real) algebraic numbers. It is well-known from Tarski's theorem that real arithmetic, the first-order theory $\langle\mathbb{R},+, \cdot\rangle$ of reals with addition and multiplication, admits quantifier elimination and hence is decidable. It follows that any real number $x$ is algebraic iff $\{x\}$ is definable in real arithmetic. We denote by $\mathbb{N}, \mathbb{Q}_{+}, \mathbb{A}_{+}, \mathbb{R}_{+}$the restrictions of $\mathbb{Z}, \mathbb{Q}, \mathbb{A}, \mathbb{R}$ to the nonnegatives.

Recall that a complete lattice is any partially ordered set $(L, \sqsubseteq)$ such that every subset $X \subseteq L$ has a least upper bound $\bigsqcup X$ and a greatest lower bound $\prod X$. The supremum $\bigsqcup L$ and the infimum $\prod L$ are respectively denoted by $\top$ and $\perp$. A function $f \in L \rightarrow L$ is monotonic if $f(x) \sqsubseteq f(y)$ for all $x \sqsubseteq y$ in $L$. It is well-known from Knaster-Tarski's theorem that any monotonic function $f \in L \rightarrow L$ has a least fix-point given by $\prod\{x \in L \mid f(x) \sqsubseteq x\}$. For any monotonic function $f \in L \rightarrow L$, we define the monotonic function $f^{*}$ in $L \rightarrow L$ by $f^{*}(x)=\prod\{y \in L \mid(x \sqcup f(y)) \sqsubseteq y\}$. In other words $f^{*}(x)$ is the least post-fix-point of $f$ greater than $x$. Observe that $f^{*}(x)=$ $x \sqcup f\left(f^{*}(x)\right)$ for every $x \in L$.

For any complete lattice $(L, \sqsubseteq)$ and any set $S$, we also denote by $\sqsubseteq$ the partial order on $S \rightarrow L$ defined as the point-wise extension of $\sqsubseteq$, i.e. $f \sqsubseteq g$ iff $f(s) \sqsubseteq g(s)$ for all $s \in S$. The partially ordered set $(S \rightarrow L, \sqsubseteq)$ is also a complete lattice, with lub $\bigsqcup$ and glb $\prod$ satisfying $(\bigsqcup F)(s)=\bigsqcup\{f(s) \mid f \in F\}$ and $\left(\prod F\right)(s)=\prod\{f(s) \mid f \in F\}$ for any subset $F \subseteq S \rightarrow L$.

For any set $S$, we write $\mathbb{P}(S)$ for the set of subsets of $S$. The partially ordered set $(\mathbb{P}(S), \subseteq)$ is a complete lattice, with lub $\bigcup$ and glb $\bigcap$. The identity function over any set $S$ is written $\mathbb{1}_{S}$, and shortly $\mathbb{1}$ when the set $S$ is clear from the context.

Let $\Sigma$ be a (potentially infinite) a set of letters. We write $\Sigma^{*}$ for the set of all (finite) sequences $l_{1} \cdots l_{k}$ over $\Sigma$, and $\varepsilon$ denotes the empty sequence. Given any two sequences $w$ and $w^{\prime}$, we denote by $w \cdot w^{\prime}$ (shortly written $w w^{\prime}$ ) their concatenation. A subset of $\Sigma^{*}$ is called a language.

\subsection{Closed convex sets and polyhedra}

We assume a fixed positive integer $n$ called the dimension. The components of a vector $\boldsymbol{x} \in \mathbb{R}^{n}$ are denoted by $\boldsymbol{x}=\left(x_{1}, \ldots, x_{n}\right)$. Operations on vectors are extended to subsets of $\mathbb{R}^{n}$ in the obvious way, e.g. $S+S^{\prime}=\left\{\boldsymbol{x}+\boldsymbol{x}^{\prime} \mid \boldsymbol{x} \in S, \boldsymbol{x}^{\prime} \in S^{\prime}\right\}$ for any $S, S^{\prime} \subseteq \mathbb{R}^{n}$. When there is no ambiguity, the singleton $\{\boldsymbol{x}\}$ is shortly written $\boldsymbol{x}$ to unclutter notation, e.g. we write $\boldsymbol{x}+S$ instead of $\{\boldsymbol{x}\}+S$. Recall that the maximum norm $\|\cdot\|_{\infty}$ on $\mathbb{R}^{n}$ is defined by $\|\boldsymbol{x}\|_{\infty}=\max \left\{\left|x_{1}\right|, \ldots,\left|x_{n}\right|\right\}$. A subset $S$ of $\mathbb{R}^{n}$ is called bounded if $\left\{\|\boldsymbol{x}\|_{\infty} \mid \boldsymbol{x} \in S\right\} \subseteq[0, b]$ for some $b \in \mathbb{R}$. The (topological) closure, interior and boundary of a subset $S$ of $\mathbb{R}^{n}$ are respectively denoted by $\operatorname{clo}(S), \operatorname{int}(S)$ and bd $(S)$. 
We now recall some notions about convex subsets of $\mathbb{R}^{n}$ (see [Sch86] for details). Recall that this class of subsets of $\mathbb{R}^{n}$ is closed under arbitrary intersection. The convex hull of any subset $S \subseteq \mathbb{R}^{n}$, written conv $(S)$, is the smallest (w.r.t. inclusion) convex set that contains $S$. Note that conv $(S)$ is closed when $S$ is finite, but this is not true in general. We devote particular attention in the sequel to closed convex subsets of $\mathbb{R}^{n}$. This class of subsets of $\mathbb{R}^{n}$ is also closed under arbitrary intersection. The closed convex hull of any subset $S \subseteq \mathbb{R}^{n}$, written cloconv $(S)$, is the smallest (w.r.t. inclusion) closed convex set that contains $S$. Remark that cloconv $(S)=\operatorname{clo}(\operatorname{conv}(S))$. For any vector $\boldsymbol{d} \in \mathbb{R}^{n}$, we define $\uparrow \boldsymbol{d}$ to be the convex set $\uparrow \boldsymbol{d}=\left\{\lambda \boldsymbol{d} \mid \lambda \in \mathbb{R}_{+}\right\}$. The recession cone $0^{+} S$ of any subset $S$ of $\mathbb{R}^{n}$ is the set of all vectors $\boldsymbol{d} \in \mathbb{R}^{n}$ such that $S+\uparrow \boldsymbol{d} \subseteq S$. Note that $\mathbf{0} \in 0^{+} S$. If $C$ is a closed convex subset of $\mathbb{R}^{n}$ then $0^{+} C$ is also closed and convex. If moreover $C$ is non-empty then for any $\boldsymbol{d} \in \mathbb{R}^{n}$, we have $\boldsymbol{d} \in 0^{+} C$ iff there exists $\boldsymbol{x} \in C$ such that $\boldsymbol{x}+\uparrow \boldsymbol{d} \subseteq C$.

Let us fix $\mathbb{E} \in\{\mathbb{Q}, \mathbb{A}, \mathbb{R}\}$. A subset $S$ of $\mathbb{R}^{n}$ is called an $\mathbb{E}$-half-space if there exists $\boldsymbol{\alpha} \in \mathbb{F}^{n} \backslash\{\boldsymbol{0}\}$ and $c \in \mathbb{F}$ such that $S=\left\{\boldsymbol{x} \in \mathbb{R}^{n} \mid \alpha_{1} x_{1}+\cdots+\alpha_{n} x_{n} \leq c\right\}$. An $\mathbb{F}$-polyhedron is any finite intersection of $\mathbb{F}$-half-spaces. In the sequel, $\mathbb{Q}$-polyhedrality (resp. A-polyhedrality, $\mathbb{R}$-polyhedrality) is also called rational polyhedrality (resp. algebraic polyhedrality, real polyhedrality). Moreover, $\mathbb{R}$-polyhedra and a $\mathbb{R}$-half-spaces are shortly called polyhedra and half-spaces. Remark that any subset of $\mathbb{R}^{n}$ is $\mathbb{A}$-polyhedral iff it is both polyhedral and definable in $\langle\mathbb{R},+, \cdot\rangle$.

The class of closed convex subsets of $\mathbb{R}^{n}$ is written $\mathcal{C}_{n}$. We denote by $\sqsubseteq$ the inclusion partial order on $\mathcal{C}_{n}$. Observe that $\left(\mathcal{C}_{n}, \sqsubseteq\right)$ is a complete lattice, with lub $\bigsqcup$ and glb $\Pi$ satisfying $\bigsqcup X=$ cloconv $(\bigcup X)$ and $\sqcap X=\bigcap X$ for any subset $X \subseteq \mathcal{C}_{n}$.

\section{Convex Acceleration for Guarded Translation Systems}

We now define the class of guarded translation systems, for which we investigate the computability of data-flow solutions in the complete lattice $\left(\mathcal{C}_{n}, \sqsubseteq\right)$. This class intuitively represents programs with real-valued variables, where conditions are closed convex sets and transformations are restricted to translations.

An $n$-dim action is any pair $(G, \boldsymbol{d})$ where $G \in \mathcal{C}_{n}$ is called the guard and $\boldsymbol{d} \in \mathbb{R}^{n}$ is called the displacement. We write $\mathcal{A}_{n}=\mathcal{C}_{n} \times \mathbb{R}^{n}$ the set of all $n$-dim actions. A trace is any finite sequence $a_{1} \cdots a_{k} \in \mathcal{A}_{n}^{*}$. The data-flow semantics $\llbracket a \rrbracket$ of any $n$-dim action $a=(G, \boldsymbol{d})$ is the monotonic function in $\mathrm{C}_{n} \rightarrow \mathcal{C}_{n}$ defined by $\llbracket a \rrbracket(C)=(G \cap C)+\boldsymbol{d}$.

An $n$-dim guarded translation system $(G T S)$ is any pair $\mathcal{S}=(\mathcal{X}, T)$ where $\mathcal{X}$ is a finite set of variables and $T \subseteq \mathcal{X} \times \mathcal{A}_{n} \times \mathcal{X}$ is a finite set of transitions. A transition $t=\left(X, a, X^{\prime}\right)$ is also written $X \stackrel{a}{\rightarrow} X^{\prime}$ or $X^{\prime}:=a(X)$, and we say that $a$ (resp. $X$, $X^{\prime}$ ) is the action (resp. input variable, output variable) of $t$. A path in $\mathcal{S}$ is any finite sequence $t_{1} \cdots t_{k} \in T^{*}$ such that the output variable of $t_{i}$ is equal to the input variable of $t_{i+1}$ for every $1 \leq i<k$. We say that a path $\pi$ is a path from $X$ to $X^{\prime}$ if either (1) $\pi=\varepsilon$ and $X=X^{\prime}$, or (2) $\pi=t_{1} \cdots t_{k}$ and $X, X^{\prime}$ respectively are the input variable of $t_{1}$ and the output variable of $t_{k}$. Any path with no repeated variable is called a simple path. A cycle is any non-empty path from some variable $X$ to $X$. Any cycle of the form $t \cdot \pi$ where $t$ is a transition and $\pi$ is a simple path is called a simple cycle. A valuation 
is any function $\rho$ in $\mathcal{X} \rightarrow \mathcal{C}_{n}$. An $n$-dim initialized guarded translation system (IGTS) is any triple $\mathcal{S}=\left(\mathcal{X}, T, \rho_{0}\right)$ where $(\mathcal{X}, T)$ is an $n$-dim GTS and $\rho_{0} \in \mathcal{X} \rightarrow \mathcal{C}_{n}$ is an initial valuation.

Intuitively, a transition $X \stackrel{a}{\rightarrow} X^{\prime}$ assigns variable $X^{\prime}$ to $a(X)$ and does not change the other variables. Formally, the data-flow semantics $\llbracket t \rrbracket$ of any transition $t=X \stackrel{a}{\rightarrow} X^{\prime}$ is the monotonic function in $\left(\mathcal{X} \rightarrow \mathcal{C}_{n}\right) \rightarrow\left(\mathcal{X} \rightarrow \mathcal{C}_{n}\right)$ defined by $\llbracket t \rrbracket(\rho)\left(X^{\prime}\right)=$ $\llbracket a \rrbracket(\rho(X))$ and $\llbracket t \rrbracket(\rho)(Y)=\rho(Y)$ for all $Y \neq X^{\prime}$. The data-flow semantics $\llbracket \cdot \rrbracket$ is extended to sequences $w$ in $\mathcal{A}_{n}^{*} \cup T^{*}$ in the obvious way: $\llbracket \varepsilon \rrbracket=\mathbb{1}$ and $\llbracket l \cdot w \rrbracket=$ $\llbracket w \rrbracket \circ \llbracket l \rrbracket$. We also extend the data-flow semantics to languages $L$ in $\mathbb{P}\left(\mathcal{A}_{n}^{*}\right) \cup \mathbb{P}\left(T^{*}\right)$ by $\llbracket L \rrbracket=\bigsqcup_{w \in L} \llbracket w \rrbracket$.

For computability reasons, we extend $\mathbb{E}$-polyhedrality, where $\mathbb{E} \in\{\mathbb{Q}, \mathbb{A}, \mathbb{R}\}$, to actions, valuations and guarded translation systems. An $n$-dim action $(G, \boldsymbol{d})$ is called $\mathbb{F}$-polyhedral if $G$ is $\mathbb{F}$-polyhedral and $\boldsymbol{d} \in \mathbb{F}^{n}$. An $n$-dim $\operatorname{GTS}(\mathcal{X}, T)$ is called $\mathbb{F}$ polyhedral if the action of every transition $t \in T$ is $\mathbb{E}$-polyhedral. A valuation $\rho$ in $\mathcal{X} \rightarrow \mathcal{C}_{n}$ is called $\mathbb{E}$-polyhedral if $\rho(X)$ is $\mathbb{F}$-polyhedral for every $X \in \mathcal{X}$. An $n$-dim IGTS $\left(\mathcal{X}, T, \rho_{0}\right)$ is called $\mathbb{E}$-polyhedral if $(\mathcal{X}, T)$ and $\rho_{0}$ are $\mathbb{F}$-polyhedral.

Example 3.1. Consider the C-style source code given on the left-hand side below and assume that the initial values of variables $z_{1}$ and $z_{2}$ satisfy $z_{1}=1$ and $-1 \leq z_{2} \leq 1$. The corresponding IGTS $\mathcal{E}$ is depicted graphically on the right-hand side below.
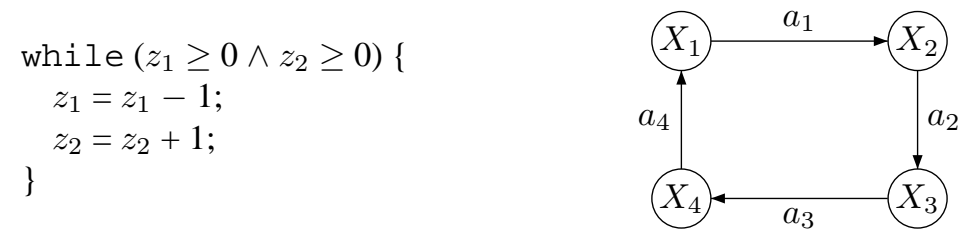

Formally, the set of variables of $\mathcal{E}$ is $\left\{X_{1}, X_{2}, X_{3}, X_{4}\right\}$, representing the values of variables $z_{1}$ and $z_{2}$ at program points $1,2,3$ and 4 . Its initial valuation is $\left\{X_{1} \mapsto\{1\} \times\right.$ $\left.[-1,1], X_{2} \mapsto \perp, X_{3} \mapsto \perp, X_{4} \mapsto \perp\right\}$, and its set of transitions is $\left\{t_{1}, t_{2}, t_{3}, t_{4}\right\}$, with:

$$
\begin{array}{ll}
t_{1}=X_{1} \stackrel{a_{1}}{\longrightarrow} X_{2}, a_{1}=\left(\mathbb{R}_{+}^{2}, \mathbf{0}\right) & t_{2}=X_{2} \stackrel{a_{2}}{\longrightarrow} X_{3}, a_{2}=\left(\mathbb{R}^{2},(-1,0)\right) \\
t_{4}=X_{4} \stackrel{a_{4}}{\longrightarrow} X_{1}, a_{4}=\left(\mathbb{R}^{2}, \mathbf{0}\right) & t_{3}=X_{3} \stackrel{a_{3}}{\longrightarrow} X_{4}, a_{3}=\left(\mathbb{R}^{2},(0,1)\right)
\end{array}
$$

Given any $n$-dim IGTS $\mathcal{S}=\left(\mathcal{X}, T, \rho_{0}\right)$, the merge over all paths solution $(M O P$ solution) of $\mathcal{S}$, written $\Pi_{\mathcal{S}}$, and the minimum fix-point solution (MFP-solution) of $\mathcal{S}$, written $\Lambda_{\mathcal{S}}$, are the valuations defined as follows:

$$
\begin{aligned}
& \Pi_{\mathcal{S}}=\bigsqcup\left\{\llbracket \pi \rrbracket\left(\rho_{0}\right) \mid \pi \in T^{*} \text { is a path }\right\} \\
& \Lambda_{\mathcal{S}}=\prod\left\{\rho \in \mathcal{X} \rightarrow \mathcal{C}_{n} \mid \rho_{0} \sqsubseteq \rho \text { and } \llbracket t \rrbracket(\rho) \sqsubseteq \rho \text { for all } t \in T\right\}
\end{aligned}
$$

Remark that for any sequence $\pi \in T^{*}$ and variable $X \in \mathcal{X}$, there exists a path $\pi^{\prime}$ such that $\llbracket \pi \rrbracket\left(\rho_{0}\right)(X)=\llbracket \pi^{\prime} \rrbracket\left(\rho_{0}\right)(X)$. Recall also that $\llbracket T \rrbracket^{*}(\rho)$ denotes the least postfix-point of $\llbracket T \rrbracket$ greater than $\rho$. Therefore it follows from the above definitions that $\Pi_{\mathcal{S}}=\llbracket T^{*} \rrbracket\left(\rho_{0}\right)$ and $\Lambda_{\mathcal{S}}=\llbracket T \rrbracket^{*}\left(\rho_{0}\right)$. 
Example 3.2. Consider the IGTS $\mathcal{E}^{\prime}=\left(\{X\},\{X \stackrel{a}{\rightarrow} X\},\left\{X \mapsto C_{0}\right\}\right)$ with $a=$ $\left(\mathbb{R}_{+}^{2},(-1,1)\right)$ and $C_{0}=\{1\} \times[-1,1]$. Intuitively $\mathcal{E}^{\prime}$ corresponds to a compact version of the IGTS $\mathcal{E}$ from Example 3.1, where the cycle is shortened into a single "selfloop" transition. The convex sets $C_{0}, \llbracket a \rrbracket\left(C_{0}\right)$ and $\llbracket a a \rrbracket\left(C_{0}\right)$ are depicted below (respectively in black, blue and red). Since $\llbracket a a a \rrbracket\left(C_{0}\right)$ is empty, we get that $\llbracket a^{*} \rrbracket\left(C_{0}\right)=$ $C_{0} \sqcup \llbracket a \rrbracket\left(C_{0}\right) \sqcup \llbracket a a \rrbracket\left(C_{0}\right)$. The characterization of $\llbracket a \rrbracket^{*}\left(C_{0}\right)$ is more complex ; the key point here is to show that the set $\{0\} \times[0,2]$ is necessarily contained $\llbracket a \rrbracket^{*}\left(C_{0}\right)$. The sets $\llbracket a^{*} \rrbracket\left(C_{0}\right)$ and $\llbracket a \rrbracket^{*}\left(C_{0}\right)$ are also depicted below.
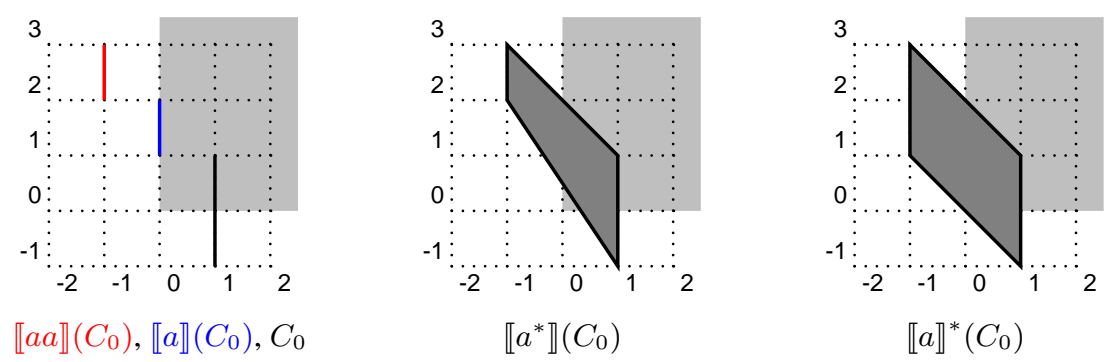

The MOP-solution $\Pi_{\mathcal{E}^{\prime}}$ and the MFP-solution $\Lambda_{\mathcal{E}^{\prime}}$ of the IGTS $\mathcal{E}^{\prime}$ are the valuations $\Pi_{\mathcal{E}^{\prime}}=\left\{X \mapsto \llbracket a^{*} \rrbracket\left(C_{0}\right)\right\}$ and $\Lambda_{\mathcal{E}^{\prime}}=\left\{X \mapsto \llbracket a \rrbracket^{*}\left(C_{0}\right)\right\}$.

Recall that our objective is to speed up, using acceleration-based techniques, the computation of the MFP-solution for initialized guarded translation systems. Recast in our setting, exact acceleration [BW94, CJ98, FIS03, FL02, BIL06] intuitively consists in computing the exact effect $\bigcup_{k \in \mathbb{N}} \llbracket\left(a_{1} \cdots a_{k}\right)^{k} \rrbracket\left(C_{0}\right)$ of some cycle $X \stackrel{a_{1}}{\longrightarrow}$ $X_{1} \cdots X_{k-1} \stackrel{a_{k}}{\longrightarrow} X$, starting with some $C_{0} \in \mathcal{C}_{n}$ in $X$. Thus we may want define acceleration as the closed convex hull of this expression. However it would be even more desirable to compute the larger set $\llbracket\left(a_{1} \cdots a_{k}\right) \rrbracket^{*}\left(C_{0}\right)$ since it is contained in the MFP-solution. We thus come to the following definition. Given any trace $\sigma$ in $\mathcal{A}_{n}^{*}$, the function $\llbracket \sigma^{*} \rrbracket$ (resp. $\llbracket \sigma \rrbracket^{*}$ ) is called the MOP-acceleration of $\sigma$ (resp. the MFPacceleration of $\sigma$ ).

As will be apparent in section 5, trace-based acceleration is not in general sufficient to guarantee termination of the Kleene fix-point iteration, even for "cyclic" IGTS. The reason is that trace-based acceleration distinguishes a variable $X$ (the "input variable" of the cycle to be accelerated) and abstracts away all other variables in the "current" valuation $\rho$ of the fix-point iteration. Hence we also introduce acceleration of cycles, where we intuitively consider the MOP-solution or MFP-solution of the system restricted to this cycle. Formally, given any simple cycle $\pi$ in $T^{*}$, the MOP-acceleration of $\pi$ (resp. the MFP-acceleration of $\pi$ ) is the function $\llbracket U^{*} \rrbracket$ (resp. $\llbracket U \rrbracket^{*}$ ) where $U$ is the set of transitions that occur in $\pi$. Note that these accelerations may be extended to arbitrary cycles through the notion of unfoldings [LS07].

The rest of the paper is devoted to the characterization and computation of these accelerations: section 4 focuses on acceleration for traces and section 5 investigates acceleration for simple cycles. 


\section{Acceleration for Traces}

We focus in this section on MOP-acceleration and MFP-acceleration for traces. Remark that for any $\sigma=a_{1} \cdots a_{k} \in \mathcal{A}_{n}^{*}$, with $a_{i}=\left(G_{i}, \boldsymbol{d}_{\boldsymbol{i}}\right)$, we have $\llbracket \sigma \rrbracket=\llbracket a_{\sigma} \rrbracket$ where $a_{\sigma}=\left(G_{\sigma}, \boldsymbol{d}_{\boldsymbol{\sigma}}\right)$ is defined by $\boldsymbol{d}_{\boldsymbol{\sigma}}=\sum_{i=1}^{k} \boldsymbol{d}_{\boldsymbol{i}}$ and $G_{\sigma}=\bigcap_{i=1}^{k}\left(G_{i}-\sum_{j=1}^{i-1} \boldsymbol{d}_{\boldsymbol{j}}\right)$. It follows that $\llbracket \sigma^{*} \rrbracket=\llbracket a_{\sigma}^{*} \rrbracket$ and $\llbracket \sigma \rrbracket^{*}=\llbracket a_{\sigma} \rrbracket^{*}$. Therefore we will w.l.o.g. restrict our attention to MOP-acceleration and MFP-acceleration for single actions.

Consider an $n$-dim action $a=(G, \boldsymbol{d})$ and a closed convex set $C_{0} \in \mathcal{C}_{n}$. Recall that $\llbracket a^{*} \rrbracket\left(C_{0}\right)=\bigsqcup_{k \in \mathbb{N}} \llbracket a^{k} \rrbracket\left(C_{0}\right)$. Observe that for every $k \in \mathbb{N}$ we have $\llbracket a^{k} \rrbracket\left(C_{0}\right)=$ $\left(G_{k} \cap C_{0}\right)+k \boldsymbol{d}$ where $G_{k}=\bigcap_{i=0}^{k-1}(G-i \boldsymbol{d})$. By convexity of $G$ we deduce that $G_{k}=G \cap(G-(k-1) d)$ for every $k \geq 1$. Hence we have :

$$
\llbracket a^{*} \rrbracket\left(C_{0}\right)=C_{0} \sqcup\left(\operatorname{cloconv}\left(G \cap\left(\left(G \cap C_{0}\right)+\mathbb{N} \boldsymbol{d}\right)\right)+\boldsymbol{d}\right)
$$

The main difficulty here lies in the computation of cloconv $\left(G \cap\left(\left(G \cap C_{0}\right)+\mathbb{N} \boldsymbol{d}\right)\right)$.

We introduce the class of poly-based semilinear sets and show that this class is closed under sum, union and intersection. We call poly-based linear any subset of $\mathbb{R}^{n}$ of the form $B+\sum_{\boldsymbol{p} \in P} \mathbb{N} \boldsymbol{p}$ where $B$ is a bounded polyhedron and $P$ is a finite subset of $\mathbb{Z}^{n}$. A poly-based semilinear set is any finite union of poly-based linear sets. Note that poly-based semilinearity generalizes standard (integer) semilinearity [GS66] in that for any subset $Z$ of $\mathbb{Z}^{n}, Z$ is semilinear iff $Z$ is poly-based semilinear.

Lemma 4.1. Every polyhedron is a poly-based linear set. Poly-based semilinear sets are closed under sum, union and intersection.

We obtain from Lemma 4.1 that $\llbracket a^{*} \rrbracket\left(C_{0}\right)=C_{0} \sqcup(\operatorname{cloconv}(S)+\boldsymbol{d})$ for some poly-based semilinear set $S$. Since cloconv $\left(\sum_{\boldsymbol{p} \in P} \mathbb{N} \boldsymbol{p}\right)=\sum_{\boldsymbol{p} \in P} \uparrow \boldsymbol{p}$ for any subset $P$ of $\mathbb{R}^{n}$, we get that cloconv $(S)$ is a polyhedron and hence we come to the following proposition.

Proposition 4.2. For any $n$-dim action $a=(G, \boldsymbol{d})$ and closed convex set $C_{0} \in \mathcal{C}_{n}$, if $G$ and $C_{0}$ are polyhedra then $\llbracket a^{*} \rrbracket\left(C_{0}\right)$ is a polyhedron.

Remark that the proof of Proposition 4.2 is constructive (since the proof of Lemma 4.1 is constructive). It follows that for each $\mathbb{E} \in\{\mathbb{Q}, \mathbb{A}\}$, the set $\llbracket a^{*} \rrbracket\left(C_{0}\right)$ is an effectively computable $\mathbb{F}$-polyhedron when $a$ and $C_{0}$ are $\mathbb{F}$-polyhedral. The following proposition gives a simple expression of the MOP-acceleration for bounded closed convex sets.

Proposition 4.3. For any $n$-dim action $a=(G, \boldsymbol{d})$ and closed convex set $C_{0} \in \mathcal{C}_{n}$, if $G \cap C_{0}$ is bounded then we have:

- if $G \cap C_{0} \neq \emptyset$ and $\boldsymbol{d} \in 0^{+} G$ then $\llbracket a^{*} \rrbracket\left(C_{0}\right)=C_{0}+\uparrow \boldsymbol{d}$, and

- otherwise $\llbracket a^{k} \rrbracket\left(C_{0}\right)=\emptyset$ for some $k \in \mathbb{N}$, and $\llbracket a^{*} \rrbracket\left(C_{0}\right)=\bigsqcup_{i=0}^{k-1} \llbracket a^{i} \rrbracket\left(C_{0}\right)$.

Our next result gives a surprisingly simple expression of the MFP-acceleration for arbitrary $n$-dim actions. 
Proposition 4.4. For any n-dim action $a=(G, d)$ and closed convex set $C_{0} \in \mathfrak{C}_{n}$, we have:

$$
\llbracket a \rrbracket^{*}\left(C_{0}\right)= \begin{cases}C_{0} & \text { if } G \cap C_{0}=\emptyset \\ C_{0} \sqcup\left(\left(G \cap\left(C_{0}+\uparrow \boldsymbol{d}\right)\right)+\boldsymbol{d}\right) & \text { otherwise }\end{cases}
$$

It follows from Proposition 4.4 that $\llbracket a \rrbracket^{*}\left(C_{0}\right)$ is a polyhedron when $G$ and $C_{0}$ are polyhedra. If moreover $a$ and $C_{0}$ are $\mathbb{F}$-polyhedral, with $\mathbb{F} \in\{\mathbb{Q}, \mathbb{A}\}$, then $\llbracket a \rrbracket^{*}\left(C_{0}\right)$ is an effectively computable $\mathbb{E}$-polyhedron.

We now compare our MFP-acceleration approach with abstract loop acceleration introduced in [GH06] as a complement to widening for linear relation analysis. Let us recast the definition of [GH06] in our setting. The abstract-acceleration $\llbracket a \rrbracket^{\otimes}$ of any $n$ $\operatorname{dim}$ action $a=(G, \boldsymbol{d})$ is the monotonic function in $\mathcal{C}_{n} \rightarrow \mathcal{C}_{n}$ defined by $\llbracket a \rrbracket^{\otimes}\left(C_{0}\right)=$ $C_{0} \sqcup$ cloconv $\left(\left\{\boldsymbol{x} \in \mathbb{R}^{n} \mid \exists \boldsymbol{x}_{\mathbf{0}} \in G \cap C_{0}, \boldsymbol{x} \in\left(\boldsymbol{x}_{\mathbf{0}}+\uparrow \boldsymbol{d}\right) \cap(G+\boldsymbol{d})\right\}\right)$. Observe that $\llbracket a \rrbracket^{\otimes}\left(C_{0}\right)=C_{0} \sqcup\left(\left(G \cap C_{0}\right)+\uparrow \boldsymbol{d}\right) \cap(G+\boldsymbol{d})$. Hence we obtain the following relationships between MOP-acceleration, MFP-acceleration and abstract-acceleration:

$$
\llbracket a^{*} \rrbracket\left(C_{0}\right) \sqsubseteq \llbracket a \rrbracket^{\otimes}\left(C_{0}\right)=C_{0} \sqcup \llbracket a \rrbracket^{*}\left(C_{0} \cap G\right) \sqsubseteq \llbracket a \rrbracket^{*}\left(C_{0}\right)
$$

Note in particular that $\llbracket a \rrbracket^{\otimes}\left(C_{0}\right)=\llbracket a \rrbracket^{*}\left(C_{0}\right)$ when $C_{0} \subseteq G$. It turns out that abstractacceleration is not sufficient to guarantee termination of the Kleene fix-point iteration even for guarded translation systems consisting in a single "self-loop" transition. Consider our running example, the IGTS given in Example 3.2, and recall that $C_{0}=\{1\} \times[-1,1]$. The sequence $\left(C_{k}\right)_{k \in \mathbb{N}}$ defined by $C_{k+1}=\llbracket a \rrbracket^{\otimes}\left(C_{k}\right)$ corresponds, for this example, to the abstract-accelerated Kleene fix-point iteration suggested in [GH06]. An induction on $k$ shows that for every $k \geq 1$, the set $C_{k}$ is the convex hull of $\left\{(1,-1),(1,1),(-1,3),\left(-1, y_{k}\right)\right\}$ where $y_{k}=1+\frac{1}{2^{k}-1}$. The first sets $C_{0}, C_{1}, C_{2}$ and $C_{3}$ of the iteration are depicted on the right (darker sets corresponds to smaller indices). It follows that the sequence $\left(C_{k}\right)_{k \in \mathbb{N}}$ is (strictly) increasing and hence this accelerated Kleene fix-point iteration does not terminate. Note that the situation would not be better with MOP-acceleration. However as already noted in Example 3.2, MFP-acceleration of $a$ directly produces the

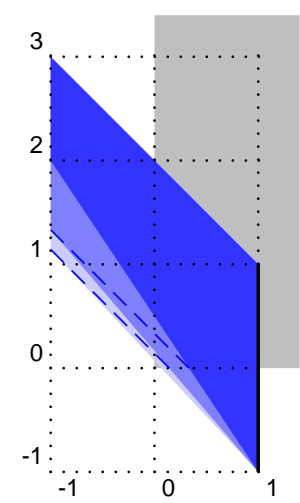
MFP-solution. Hence the MFP-accelerated Kleene fix-point iteration would reach the fix-point after just one iteration. Notice that MFP-acceleration and abstract-acceleration have the same computational complexity.

\section{Acceleration for cycles}

We investigate the computation of the MOP-acceleration (resp. the MFP-acceleration) of a simple cycle. Following our definitions, this problem reduces to the computation of the MOP-solution (resp. the MFP-solution) of an IGTS that contains all its transitions into a unique (up to permutations) simple cycle $\pi=X_{1} \stackrel{a_{1}}{\longrightarrow} \cdots X_{k} \stackrel{a_{k}}{\longrightarrow} X_{1}$, called cyclic. We only consider the MFP-solution computation in the sequel since the following equality shows that the MOP-solution of a cyclic IGTS reduces to the computation 
of the MOP-acceleration of the trace $\sigma=a_{1} \ldots a_{k}$ :

$$
\Pi_{\mathcal{S}}\left(X_{1}\right)=\bigsqcup_{i=1}^{k} \llbracket \sigma^{*} \rrbracket \circ \llbracket a_{i+1} \ldots a_{k} \rrbracket\left(\rho_{0}\left(X_{i}\right)\right)
$$

We first explain why the previous reduction cannot be extended to the MFP-solution. Naturally, when the initial valuation $\rho_{0}$ satisfies $\rho_{0}(X)=\perp$ for all but one variable $X_{i}$, the following equality shows that the MFP-solution reduces to the MFP-acceleration of traces (values of $\Lambda_{\mathfrak{s}}$ in $X_{2}, \ldots, X_{k}$ are obtained by circular permutations):

$$
\Lambda_{\mathcal{S}}\left(X_{1}\right)=\llbracket \sigma \rrbracket^{*} \circ \llbracket a_{i+1} \ldots a_{k} \rrbracket\left(\rho_{0}\left(X_{i}\right)\right)
$$

However, this case is not sufficient since we want to apply MFP-acceleration an an point during an iterative computation of MFP-solutions. The 2-dim cyclic rational polyhedral IGTS $\mathcal{E}_{2}$ formally defined below shows that the MFP-solution $\Lambda_{\mathcal{S}}$ cannot be reduced to MFP-acceleration of traces for a general initial valuation $\rho_{0}$. In fact, we prove in the sequel that the MFP-solution of $\mathcal{E}_{2}$ is $\mathbb{A}$-polyhedral but not $\mathbb{Q}$-polyhedral. Since MFP-accelerations of traces only produce $\mathbb{Q}$-polyhedral valuations we deduce that the MFP-solution cannot be obtained using MFP-acceleration of traces.

Example 5.1. Consider the cyclic 2-dim IGTS $\mathcal{E}_{2}$ depicted graphically on the left-hand side below.
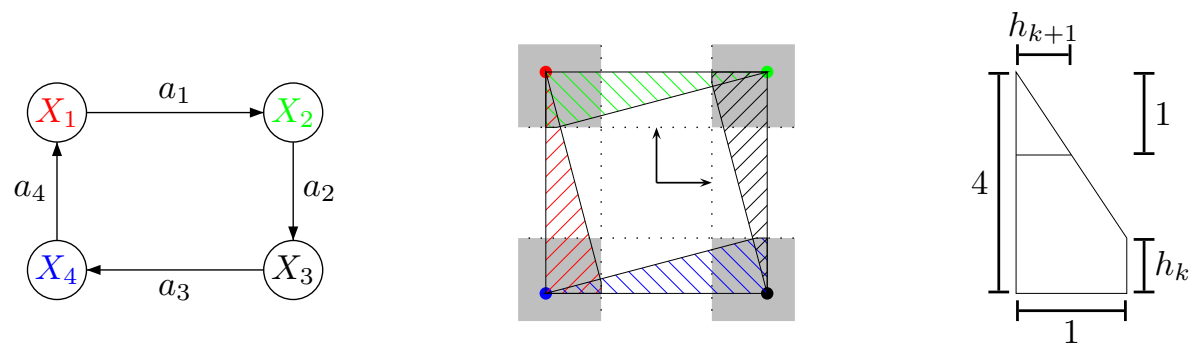

Formally the initial valuation $\rho_{0}$ of $\mathcal{E}_{2}$ is $\left\{X_{1} \mapsto\{(-2,2)\}, X_{2} \mapsto\{(2,2)\}, X_{3} \mapsto\right.$ $\left.\{(2,-2)\}, X_{4} \mapsto\{(-2,-2)\}\right\}$, and its actions $a_{1}=\left(G_{1}, \mathbf{0}\right), a_{2}=\left(G_{2}, \mathbf{0}\right), a_{3}=$ $\left(G_{3}, \mathbf{0}\right), a_{4}=\left(G_{4}, \mathbf{0}\right)$ are defined by $\left.\left.G_{1}=\right]-\infty,-1\right] \times\left[1,+\infty\left[, G_{2}=[1,+\infty[\times\right.\right.$ $\left[1,+\infty\left[, G_{3}=[1,+\infty[\times]-\infty,-1]\right.\right.$ and $\left.\left.\left.\left.G_{4}=\right]-\infty,-1\right] \times\right]-\infty,-1\right]$.

The MFP-solution of the IGTS $\varepsilon_{2}$ can be obtained by first proving that the Kleene iteration $(\mathbb{1} \sqcup \llbracket T \rrbracket)^{k+2}\left(\rho_{0}\right)$ is equal to the valuation $\Lambda_{\mathcal{E}_{2}, h_{k}}$ (The values of $\Lambda_{\mathcal{E}_{2}, h}$ in $X_{1}, X_{2}, X_{3}, X_{4}$ are graphically pictured in red, green, black and blue in the center of the previous figure) where $\Lambda_{\varepsilon_{2}, h}$ is the following valuation parameterized by a real number $h$ and where $\left(h_{k}\right)_{k \geq 0}$ is the sequence of rational numbers defined by $h_{0}=0$ and $h_{k+1}=\frac{1}{4-h_{k}}$ (this last equality can be geometrically obtained from the right-hand side picture of the previous figure).

$$
\begin{aligned}
& \Lambda_{\mathcal{E}_{2}, h}\left(X_{1}\right)=\operatorname{conv}(\{(-2,2),(-2,-2),(-1,-2),(-1,-2+h)\}) \\
& \Lambda_{\mathcal{E}_{2}, h}\left(X_{2}\right)=\operatorname{conv}(\{(2,2),(-2,2),(-2,1),(-2+h, 1)\}) \\
& \Lambda_{\mathcal{E}_{2}, h}\left(X_{3}\right)=\operatorname{conv}(\{(2,-2),(2,2),(1,2),(1,2-h)\}) \\
& \Lambda_{\mathcal{E}_{2}, h}\left(X_{4}\right)=\operatorname{conv}(\{(-2,-2),(2,-2),(2,-1),(2-h,-1)\})
\end{aligned}
$$


Lemma 5.2. We have $(\mathbb{1} \sqcup \llbracket T \rrbracket)\left(\Lambda_{\mathcal{E}_{2}, h}\right)=\Lambda_{\mathcal{E}_{2}, \frac{1}{4-h}}$ for any $0 \leq h \leq 2-\sqrt{3}$.

As $\Lambda_{\varepsilon_{2}, 0}=(\mathbb{1} \sqcup \llbracket T \rrbracket)^{2}\left(\rho_{0}\right)$ we deduce that $\Lambda_{\varepsilon_{2}, h_{k}}=(\mathbb{1} \sqcup \llbracket T \rrbracket)^{k+2}\left(\rho_{0}\right)$ for any $k \geq 0$ from the previous lemma 5.2.

Lemma 5.3. The sequence $\left(h_{k}\right)_{k \geq 0}$ converges to the algebraic number $2-\sqrt{3}$.

Since $\Lambda_{\mathcal{\varepsilon}_{2}, h_{k}} \sqsubseteq \Lambda_{\mathcal{E}_{2}}$, we deduce from lemma 5.3 that $\Lambda_{\mathcal{E}_{2,2-\sqrt{3}}} \sqsubseteq \Lambda_{\mathcal{\varepsilon}_{2}}$. Observe that lemma 5.2 proves that $\Lambda_{\varepsilon_{2}, 2-\sqrt{3}}$ is a post-fix-point. Thus $\Lambda_{\mathcal{E}_{2}, 2-\sqrt{3}}$ is the MFPsolution. Note that this valuation is $\mathbb{A}$-polyhedral but not $\mathbb{Q}$-polyhedral. We will actually show in the next section that the MFP-solution of any 2-dim A-polyhedral IGTS (not necessarily cyclic) is A-polyhedral.

Now we provide an example of 3 -dim cyclic $\mathbb{Q}$-polyhedral IGTS $\varepsilon_{3}$ corresponding to a slightly modified version of $\mathcal{E}_{2}$ that exhibits a non-polyhedral MFP-solution.

Example 5.4. Consider the cyclic 3 -dim IGTS $\varepsilon_{3}$ formally defined as $\varepsilon_{2}$ except for (a) its initial valuation $\rho_{0}$ equal to $\left\{X_{1} \mapsto(-1,1,0)+\uparrow \boldsymbol{e}_{3}, X_{2} \mapsto(1,1,0)+\uparrow \boldsymbol{e}_{\mathbf{3}}, X_{3} \mapsto\right.$ $\left.(1,-1,0)+\uparrow e_{3}, X_{4} \mapsto(-1,-1,0)+\uparrow e_{3}\right\}$ where $e_{3}=(0,0,1)$, and (b) its actions $a_{1}, a_{2}, a_{3}, a_{4}$ defined as follows ( $\mathbb{R}_{-}$is the set of non-positive real numbers $\left.-\mathbb{R}_{+}\right)$:

$$
\begin{array}{ll}
a_{1}=\left(\mathbb{R}_{-} \times \mathbb{R}_{+} \times \mathbb{R}, \boldsymbol{e}_{\mathbf{3}}\right) & a_{2}=\left(\mathbb{R}_{+} \times \mathbb{R}_{+} \times \mathbb{R}, \boldsymbol{e}_{\mathbf{3}}\right) \\
a_{4}=\left(\mathbb{R}_{-} \times \mathbb{R}_{-} \times \mathbb{R}, \boldsymbol{e}_{\mathbf{3}}\right) & a_{3}=\left(\mathbb{R}_{+} \times \mathbb{R}_{-} \times \mathbb{R}, \boldsymbol{e}_{\mathbf{3}}\right)
\end{array}
$$

Let us denote by $\Lambda_{\varepsilon_{3}, k}$ for any $k \in\{2, \ldots,+\infty\}$ the following valuation where $h_{i}=\frac{1}{i}$ for $i \geq 1,\left(z_{i}\right)_{i \geq 1}$ is defined by the initial value $z_{1}=\frac{3}{2}$ and the induction $z_{i+1} \stackrel{i}{=}$ $1+z_{i \cdot} \cdot \frac{i}{i+1}$, and $\boldsymbol{e}_{\mathbf{3}}=(0,0,1)$.

$\Lambda_{\varepsilon_{3}, k}\left(X_{1}\right)=\operatorname{conv}\left(\{(-1,1,0),(-1,-1,1)\} \cup\left\{\left(0,-h_{i}, z_{i}\right) \mid 1 \leq i<k\right\}\right)+\uparrow \boldsymbol{e}_{\mathbf{3}}$ $\Lambda_{\varepsilon_{3}, k}\left(X_{2}\right)=\operatorname{conv}\left(\{(1,1,0) \quad,(-1,1,1)\} \cup\left\{\left(-h_{i}, 0, z_{i}\right) \mid 1 \leq i<k\right\}\right)+\uparrow \boldsymbol{e}_{\mathbf{3}}$ $\Lambda_{\mathcal{E}_{3}, k}\left(X_{3}\right)=\operatorname{conv}\left(\{(1,-1,0),(1,1,1)\} \cup\left\{\left(0, h_{i}, z_{i}\right) \mid 1 \leq i<k\right\}\right)+\uparrow \boldsymbol{e}_{\mathbf{3}}$ $\Lambda_{\varepsilon_{3}, k}\left(X_{4}\right)=\operatorname{conv}\left(\{(-1,-1,0),(1,-1,1)\} \cup\left\{\left(h_{i}, 0, z_{i}\right) \mid 1 \leq i<k\right\}\right)+\uparrow e_{3}$

Lemma 5.5. Values of $\Lambda_{\varepsilon_{3},+\infty}$ in $X_{1}, X_{2}, X_{3}, X_{4}$ are closed convex sets but they are not polyhedral.

Since $(\mathbb{1} \sqcup \llbracket T \rrbracket)^{2}\left(\rho_{0}\right)=\Lambda_{\varepsilon_{3}, 2}$, the following lemma 5.6 proves that $(\mathbb{1} \sqcup \llbracket T \rrbracket)^{k}\left(\rho_{0}\right)=$ $\Lambda_{\mathcal{E}_{3}, k}$ for any $k \in\{2, \ldots,+\infty\}$.

Lemma 5.6. We have $(\mathbb{1} \sqcup \llbracket T \rrbracket)\left(\Lambda_{\varepsilon_{3}, k}\right)=\Lambda_{\varepsilon_{3}, k+1}$ for any $k \in\{2, \ldots,+\infty\}$.

We deduce that $\Lambda_{\varepsilon_{3},+\infty}$ is the MFP-solution of $\varepsilon_{3}$.

Theorem 5.7. There exists a 3 -dim cyclic rational polyhedral IGTS with a MFP-solution that is not polyhedral.

\section{MFP-solution in Dimension $\leq 2$}

We have proved in the previous section that the MFP-solution of a 2-dim cyclic rational polyhedral IGTS may be not rational. In this section the MFP-solution of any 2-dim $\mathbb{E}$-polyhedral IGTS (not necessary cyclic) is proved $\mathbb{F}$-polyhedral for any $\mathbb{E} \in\{A, \mathbb{R}\}$. 
Remark 6.1. In [SW05, LS07] the 1-dim case is fully studied.

Let us first consider any $n$-dim action $a=(G, \boldsymbol{d})$, a set $S \subseteq \mathbb{R}^{n}$ and observe that the inclusion cloconv $((G \cap S)+\boldsymbol{d}) \sqsubseteq(G \cap$ cloconv $(S))+\boldsymbol{d}$ is strict in general. Nevertheless, the following lemma provides a sufficient condition to obtain the equality. Recall that bd $(G)$ is the boundary of $G$.

Lemma 6.2. We have cloconv $((G \cap S)+\boldsymbol{d})=(G \cap$ cloconv $(S))+\boldsymbol{d}$ for any $n$-dim action $a=(G, d)$ and for any set $S \subseteq \mathbb{R}^{n}$ such that $\operatorname{bd}(G) \cap \operatorname{cloconv}(S) \subseteq S$.

Let $\mathcal{S}=\left(\mathcal{X}, T, \rho_{0}\right)$ be any $n$-dim polyhedral IGTS and let $\Delta_{\mathcal{S}}$ be the following valuation :

$$
\Delta_{\mathcal{S}}(X)=\rho_{0}(X) \sqcup \bigsqcup\left\{\operatorname{bd}(G) \cap \Lambda_{\mathcal{S}}(X) \mid X \stackrel{a=(G, \boldsymbol{d})}{\longrightarrow} X^{\prime}\right\}
$$

Observe that $\Delta_{\mathcal{S}}$ is an intermediate valuation $\rho_{0} \sqsubseteq \Delta_{\mathcal{S}} \sqsubseteq \Lambda_{\mathcal{S}}$. Let us denote by $L_{X, X_{0}}$ (resp. $L_{X_{0}, X}^{E}$ ) the set of traces $\sigma$ that label some path (resp. simple path) $X_{0} \stackrel{\sigma}{\rightarrow} X$. Let $\Lambda_{\mathcal{S}}^{\prime}$ be the valuation defined by $\Lambda_{\mathcal{S}}^{\prime}(X)=$ cloconv $(S(X))$ where $S(X)$ is the following set :

$$
S(X)=\bigcup\left\{\llbracket \sigma \rrbracket\left(\Delta_{\mathcal{S}}\left(X_{0}\right)\right) \mid X_{0} \in \mathcal{X}, \sigma \in L_{X_{0}, X}\right\}
$$

Note that $S(X)$ satisfies lemma 6.2 , we deduce that $\Lambda_{\mathcal{S}}^{\prime}$ is a post-fix-point, i.e. $\llbracket T \rrbracket\left(\Lambda_{\mathcal{S}}^{\prime}\right) \sqsubseteq$ $\Lambda_{\mathcal{S}}^{\prime}$. Moreover, as $\Lambda_{\mathcal{S}}^{\prime} \sqsubseteq \Lambda_{\mathcal{S}}$ we get the equality $\Lambda_{\mathcal{S}}^{\prime}=\Lambda_{\mathcal{S}}$.

Lemma 6.3. We have the following equality :

$$
\Lambda_{\mathcal{S}}(X)=\bigsqcup\left\{\llbracket \sigma \rrbracket\left(\Delta_{\mathcal{S}}\left(X_{0}\right)\right) \mid X_{0} \in \mathcal{X}, \sigma \in L_{X_{0}, X}^{E}\right\}+0^{+} \Lambda_{\mathcal{S}}(X)
$$

We now focus on dimension 2 and assume that $\mathcal{S}$ is a 2 -dim polyhedral IGTS. Since a polyhedron is a finite (eventually empty) intersection of half-spaces, by adding some new extra variables to the IGTS, we may assume without loss of generality that all guards are either half-spaces or the whole set $\mathbb{R}^{2}$. Note that the boundary of an halfspace $\left\{x \in \mathbb{R}^{n} \mid \alpha_{1} . x_{1}+\alpha_{2} . x_{2} \leq c\right\}$ is the line $\left\{x \in \mathbb{R}^{n} \mid \alpha_{1} . x_{1}+\alpha_{2} . x_{2}=c\right\}$, and the boundary of $\mathbb{R}^{2}$ is the empty-set. Thus bd $(G) \cap \Lambda_{\mathcal{S}}(X)$ is polyhedral for any guard $G$ and any variable $X$. We deduce that $\Delta_{\mathcal{S}}$ is polyhedral. Moreover, as 2-dim closed convex cones are polyhedral we deduce that $0^{+} \Lambda_{\mathcal{S}}(X)$ is polyhedral for any variable $X$. We have proved the following theorem.

Theorem 6.4. The MFP-solution of any 2-dim polyhedral IGTS is polyhedral.

Finally, assume that the 2-dim IGTS $\mathcal{S}$ is a $\mathbb{A}$-polyhedral and observe that for any variable $X \in \mathcal{X}$ and for any transition $X \stackrel{a}{\rightarrow} X^{\prime}$ with $a=(G, d)$, there exists:

- three vectors $\boldsymbol{d}_{\mathbf{1}}, \boldsymbol{d}_{\mathbf{2}}, \boldsymbol{d}_{\mathbf{3}} \in \mathbb{R}^{2}$ such that $0^{+} \Lambda_{\mathcal{S}}(X)=\uparrow \boldsymbol{d}_{\mathbf{1}}+\uparrow \boldsymbol{d}_{\mathbf{2}}+\uparrow \boldsymbol{d}_{\mathbf{3}}$.

- two half-spaces $H_{1}, H_{2}$ such that bd $(G) \cap \Lambda_{\mathcal{S}}(X)=\operatorname{bd}(G) \cap H_{1} \cap H_{2}$.

Since any vector (resp. any half-space) can be defined with 2 reals (resp. 3 reals), we may constructively deduce from lemma 6.3 a formula in $\langle\mathbb{R},+, \cdot\rangle$ defining $\Lambda_{\mathcal{S}}$.

Theorem 6.5. The MFP-solution of any 2-dim A-polyhedral IGTS is effectively A-polyhedral. 


\section{References}

[BHRZ05] R. Bagnara, P. M. Hill, E. Ricci, and E. Zaffanella. Precise widening operators for convex polyhedra. Science of Computer Programming, 58(1-2):28-56, 2005.

[BIL06] M. Bozga, R. Iosif, and Y. Lakhnech. Flat parametric counter automata. In Proc. 33 rd Int. Coll. Automata, Languages, and Programming (ICALP'06), Venice, Italy, July 2006, volume 4052 of LNCS, pages 577-588. Springer, 2006.

[BW94] B. Boigelot and P. Wolper. Symbolic verification with periodic sets. In Proc. 6th Int. Conf. Computer Aided Verification (CAV'94), Stanford, CA, USA, June 1994, volume 818 of $L N C S$, pages 55-67. Springer, 1994.

[CC77] P. Cousot and R. Cousot. Abstract interpretation: A unified lattice model for static analysis of programs by construction or approximation of fixpoints. In Proc. 4th ACM Symp. Principles of Programming Languages, Los Angeles, CA, USA, pages 238-252. ACM Press, 1977.

[CC92] P. Cousot and R. Cousot. Comparing the Galois connection and widening/narrowing approaches to abstract interpretation. In Proc. 4th Int. Symp. Programming Language Implementation and Logic Programming (PLILP'92), Leuven, Belgium, Aug. 1992, volume 631 of LNCS, pages 269-295. Springer, 1992.

[CH78] P. Cousot and N. Halbwachs. Automatic discovery of linear restraints among variables of a program. In Proc. 5th ACM Symp. Principles of Programming Languages, Tucson, AZ, USA, pages 84-96. ACM Press, 1978.

[CJ98] H. Comon and Y. Jurski. Multiple counters automata, safety analysis and Presburger arithmetic. In Proc. 10th Int. Conf. Computer Aided Verification (CAV'98), Vancouver, BC, Canada, June-July 1998, volume 1427 of LNCS, pages 268-279. Springer, 1998.

[FIS03] A. Finkel, S. P. Iyer, and G. Sutre. Well-abstracted transition systems: Application to FIFO automata. Information and Computation, 181(1):1-31, 2003.

[FL02] A. Finkel and J. Leroux. How to compose Presburger-accelerations: Applications to broadcast protocols. In Proc. 22nd Conf. Found. of Software Technology and Theor. Comp. Sci. (FST\&TCS'2002), Kanpur, India, Dec. 2002, volume 2556 of LNCS, pages 145-156. Springer, 2002.

[GH06] L. Gonnord and N. Halbwachs. Combining widening and acceleration in linear relation analysis. In Proc. Static Analysis, 13th Int. Symp. (SAS'06), Seoul, Korea, Aug. 2006, volume 4134 of LNCS, pages 144-160. Springer, 2006.

[GS66] S. Ginsburg and E. H. Spanier. Semigroups, Presburger formulas and languages. Pacific J. Math., 16(2):285-296, 1966.

[Kar76] M. Karr. Affine relationship among variables of a program. Acta Informatica, 6:133-141, 1976.

[LS07] J. Leroux and G. Sutre. Accelerated data-flow analysis. In Proc. 14th Int. Static Analysis Symposium (SAS'07), Kongens Lyngby, Denmark, Aug. 2007, volume 4634 of $L N C S$, pages 184-199. Springer, 2007.

[Min01] A. Miné. A new numerical abstract domain based on difference-bound matrices. In Proc. 2nd Symp. Programs as Data Objects (PADO'01), Aarhus, Denmark, May 2001, volume 2053 of $L N C S$, pages 155-172. Springer, 2001.

[Sch86] A. Schrijver. Theory of Linear and Integer Programming. Wiley, 1986.

[SSM04] S. Sankaranarayanan, H.B. Sipma, and Z. Manna. Constraint-based linear-relations analysis. In Proc. 11th Int. Symp. Static Analysis (SAS'04), Verona, Italy, Aug. 2004, volume 3148 of LNCS, pages 53-68. Springer, 2004.

[SW05] Z. Su and D. Wagner. A class of polynomially solvable range constraints for interval analysis without widenings. Theoretical Computer Science, 345(1):122-138, 2005. 


\section{A Proof of Lemma 4.1}

We need some background material on semilinear subsets of $\mathbb{Z}^{n}$ in order to prove the lemma. A subset $Z$ of $\mathbb{Z}^{n}$ is called linear if $Z=\boldsymbol{b}+\sum_{\boldsymbol{p} \in P} \mathbb{N} \boldsymbol{p}$ for some vector $\boldsymbol{b}$ in $\mathbb{Z}^{n}$ and some finite subset $P$ of $\mathbb{Z}^{n}$. A semilinear subset of $\mathbb{Z}^{n}$ is any finite union of linear subsets of $\mathbb{Z}^{n}$. Let us recall that semilinear subsets of $\mathbb{Z}^{n}$ are precisely the subsets of $\mathbb{Z}^{n}$ that are definable in Presburger arithmetic, the first-order additive theory of the integers [GS66]. Observe that a poly-based linear set is any subset of $\mathbb{R}^{n}$ of the form $B+Z$ where $B$ is a bounded polyhedron and $Z$ is a linear subset of $\mathbb{Z}^{n}$.

Lemma 4.1. Every polyhedron is a poly-based linear set. Poly-based semilinear sets are closed under sum, union and intersection.

Proof. Consider a polyhedron $C$ contained in $\mathbb{R}^{n}$. It is well-known (see for instance [Sch86, pp. 88-89]) that $C$ may be written as $C=B+\sum_{\boldsymbol{d} \in D} \uparrow \boldsymbol{d}$ where $B$ is a bounded polyhedron and $D$ is a finite subset of $\mathbb{R}^{n}$. Let $D_{0}$ denote the bounded polyhedron $D_{0}=\left\{\sum_{\boldsymbol{d} \in D} \lambda_{\boldsymbol{d}} \boldsymbol{d} \mid \lambda_{\boldsymbol{d}} \in[0,1]\right\}$ and observe that $C=B+D_{0}+\sum_{\boldsymbol{d} \in D} \mathbb{N} \boldsymbol{d}$. We obtain that $C$ is a poly-based linear set since $B+D_{0}$ is bounded.

Closure under union of poly-based semilinear sets is immediate. Closure under sum comes from (1) distributivity of sum over union and (2) closure under sum of bounded polyhedra. Let us prove closure under intersection. From distributivity of intersection over union, it is sufficient to prove that the intersection of any two poly-based linear sets is a poly-based semilinear set. Consider two bounded polyhedra $B_{1}, B_{2}$ and two finite subsets $P_{1}, P_{2}$ of $\mathbb{Z}^{n}$, and let us write $C_{1}=B_{1}+\sum_{\boldsymbol{p} \in P_{1}} \mathbb{N} \boldsymbol{p}$ and $C_{2}=B_{2}+$ $\sum_{\boldsymbol{p} \in P_{2}} \mathbb{N} \boldsymbol{p}$. Let us define the following sets for every $h \in\{1,2\}$ and $\boldsymbol{v} \in \mathbb{Z}^{n}$ :

$$
\begin{aligned}
E_{h}^{\boldsymbol{v}} & =\left(B_{h}+(-\boldsymbol{v})\right) \cap[0,1]^{n} \\
F_{h}^{\boldsymbol{v}} & =\left(B_{h}+(-\boldsymbol{v})\right) \cap\left[0,1\left[^{n}\right.\right. \\
L_{h}^{\boldsymbol{v}} & =\boldsymbol{v}+\sum_{\boldsymbol{p} \in P_{h}} \mathbb{N} \boldsymbol{p}
\end{aligned}
$$

Note that $E_{h}^{v}$ is a bounded polyhedron and that $L_{h}^{v}$ is a linear subset of $\mathbb{Z}^{n}$. We derive from the above definitions that $B_{h}=\bigcup_{\boldsymbol{v} \in \mathbb{Z}^{n}} E_{h}^{\boldsymbol{v}}+\boldsymbol{v}=\bigcup_{\boldsymbol{v} \in \mathbb{Z}^{n}} F_{h}^{\boldsymbol{v}}+\boldsymbol{v}$ for each $h \in\{1,2\}$. The set $V_{h}=\left\{\boldsymbol{v} \in \mathbb{Z}^{n} \mid E_{h}^{\boldsymbol{v}} \neq \emptyset\right\}$ is necessarily finite since $B_{h}$ is bounded. Since $E_{h}^{\boldsymbol{v}}=F_{h}^{\boldsymbol{v}}=\emptyset$ for every $\boldsymbol{v} \in \mathbb{Z}^{n} \backslash V_{h}$, we obtain that:

$$
C_{h}=\bigcup_{\boldsymbol{v} \in V_{h}} E_{h}^{\boldsymbol{v}}+L_{h}^{\boldsymbol{v}}=\bigcup_{\boldsymbol{v} \in V_{h}} F_{h}^{\boldsymbol{v}}+L_{h}^{\boldsymbol{v}}
$$

for each $h \in\{1,2\}$. Observe that:

i) $\left(U_{1}+Z_{1}\right) \cap\left(U_{2}+Z_{2}\right) \supseteq\left(U_{1} \cap U_{2}\right)+\left(Z_{1} \cap Z_{2}\right)$ for any $U_{1}, U_{2}, Z_{1}, Z_{2} \subseteq \mathbb{R}^{n}$.

ii) This inclusion becomes an equality when $U_{1}, U_{2} \subseteq\left[0,1\left[{ }^{n}\right.\right.$ and $Z_{1}, Z_{2} \subseteq \mathbb{Z}^{n}$. 
Therefore, we get that:

$$
\begin{aligned}
C_{1} \cap C_{2} & \subseteq\left(\bigcup_{v_{1} \in V_{1}} F_{1}^{\boldsymbol{v}_{1}}+L_{1}^{\boldsymbol{v}_{1}}\right) \cap\left(\bigcup_{v_{2} \in V_{2}} F_{2}^{\boldsymbol{v}_{2}}+L_{2}^{\boldsymbol{v}_{2}}\right) \\
& \subseteq \bigcup_{\left(v_{1}, v_{2}\right) \in V_{1} \times V_{2}}\left(F_{1}^{\boldsymbol{v}_{1}}+L_{1}^{\boldsymbol{v}_{1}}\right) \cap\left(F_{2}^{\boldsymbol{v}_{2}}+L_{2}^{\boldsymbol{v}_{2}}\right) \\
& \subseteq \bigcup_{\left(v_{1}, v_{2}\right) \in V_{1} \times V_{2}}\left(F_{1}^{\boldsymbol{v}_{1}} \cap F_{2}^{\boldsymbol{v}_{2}}\right)+\left(L_{1}^{\boldsymbol{v}_{1}} \cap L_{2}^{\boldsymbol{v}_{2}}\right) \\
& \subseteq \bigcup_{\left(v_{1}, v_{2}\right) \in V_{1} \times V_{2}}\left(E_{1}^{\boldsymbol{v}_{1}} \cap E_{2}^{\boldsymbol{v}_{2}}\right)+\left(L_{1}^{\boldsymbol{v}_{1}} \cap L_{2}^{\boldsymbol{v}_{2}}\right) \\
& \subseteq \bigcup_{\left(v_{1}, v_{2}\right) \in V_{1} \times V_{2}}\left(E_{1}^{\boldsymbol{v}_{1}}+L_{1}^{\boldsymbol{v}_{1}}\right) \cap\left(E_{2}^{\boldsymbol{v}_{2}}+L_{2}^{\boldsymbol{v}_{2}}\right) \\
& \subseteq\left(\bigcup_{v_{1} \in V_{1}} E_{1}^{\boldsymbol{v}_{1}}+L_{1}^{\boldsymbol{v}_{1}}\right) \cap\left(\bigcup_{v_{2} \in V_{2}} E_{2}^{\boldsymbol{v}_{2}}+L_{2}^{\boldsymbol{v}_{2}}\right) \subseteq C_{1} \cap C_{2}
\end{aligned}
$$

Thus we come to $C_{1} \cap C_{2}=\bigcup_{\left(v_{1}, v_{2}\right) \in V_{1} \times V_{2}}\left(E_{1}^{\boldsymbol{v}_{1}} \cap E_{2}^{\boldsymbol{v}_{\mathbf{2}}}\right)+\left(L_{1}^{\boldsymbol{v}_{1}} \cap L_{2}^{\boldsymbol{v}_{\mathbf{2}}}\right)$. Remark that $E_{1}^{\boldsymbol{v}_{1}} \cap E_{2}^{\boldsymbol{v}_{2}}$ is a bounded polyhedron for every $v_{1} \in V_{1}$ and $v_{2} \in V_{2}$. Since semilinear subsets of $\mathbb{Z}^{n}$ are closed under intersection, we also get that $L_{1}^{\boldsymbol{v}_{1}} \cap L_{2}^{\boldsymbol{v}_{2}}$ is a finite union of linear subsets of $\mathbb{Z}^{n}$. We conclude that $C_{1} \cap C_{2}$ is a poly-based semilinear set.

\section{B Proof of Proposition 4.3}

Proposition 4.3. For any n-dim action $a=(G, d)$ and for any closed convex set $C_{0} \in$ $\mathcal{C}_{n}$, if $G \cap C_{0}$ is bounded then we have:

- if $G \cap C_{0} \neq \emptyset$ and $\boldsymbol{d} \in 0^{+} G$ then $\llbracket a^{*} \rrbracket\left(C_{0}\right)=C_{0}+\uparrow \boldsymbol{d}$, and

- otherwise $\llbracket a^{k} \rrbracket\left(C_{0}\right)=\emptyset$ for some $k \in \mathbb{N}$, and $\llbracket a^{*} \rrbracket\left(C_{0}\right)=\bigsqcup_{i=0}^{k-1} \llbracket a^{i} \rrbracket\left(C_{0}\right)$.

Proof. Recall that $\llbracket a^{*} \rrbracket\left(C_{0}\right)=\bigsqcup_{k \in \mathbb{N}} \llbracket a^{k} \rrbracket\left(C_{0}\right)$ and that for every $k \in \mathbb{N}$, we have $\llbracket a^{k} \rrbracket\left(C_{0}\right)=\left(G_{k} \cap C_{0}\right)+k \boldsymbol{d}$ where $G_{k}=\bigcap_{i=0}^{k-1} G-i \boldsymbol{d}$. We obtain that $\llbracket a^{k} \rrbracket\left(C_{0}\right) \sqsubseteq$ $C_{0}+\uparrow \boldsymbol{d}$ for every $k \in \mathbb{N}$, and therefore $\llbracket a^{*} \rrbracket\left(C_{0}\right) \sqsubseteq C_{0}+\uparrow \boldsymbol{d}$. Now assume that $G \cap C_{0} \neq \emptyset$ and $\boldsymbol{d} \in 0^{+} G$, and let us pick some $x \in G \cap C_{0}$. Since $x+\uparrow d \sqsubseteq G$ we get that $x \in G-k \boldsymbol{d}$ for every $k \in \mathbb{N}$. Hence $x+k \boldsymbol{d} \in \llbracket a^{k} \rrbracket\left(C_{0}\right) \sqsubseteq \llbracket a^{*} \rrbracket\left(C_{0}\right)$ for every $k \in \mathbb{N}$, and it follows by convexity of $\llbracket a^{*} \rrbracket\left(C_{0}\right)$ that $x+\uparrow \boldsymbol{d} \sqsubseteq \llbracket a^{*} \rrbracket\left(C_{0}\right)$. We deduce that $\boldsymbol{d} \in 0^{+} \llbracket a^{*} \rrbracket\left(C_{0}\right)$ and thus we come to $C_{0}+\uparrow \boldsymbol{d} \sqsubseteq \llbracket a^{*} \rrbracket\left(C_{0}\right)+\uparrow \boldsymbol{d} \sqsubseteq \llbracket a^{*} \rrbracket\left(C_{0}\right)$, which concludes the proof of the first assertion.

Observe that $\llbracket a \rrbracket\left(C_{0}\right)=\emptyset$ if $G \cap C_{0}=\emptyset$, and hence the second assertion is trivially satisfied when $G \cap C_{0}=\emptyset$. Let us now assume that $G \cap C_{0} \neq \emptyset$ and $\boldsymbol{d} \notin 0^{+} G$. Observe that $G_{k} \sqsupseteq G_{k+1}$ for every $k \in \mathbb{N}$. Since $\boldsymbol{d} \notin 0^{+} G$ we get that $\bigcap_{k \in \mathbb{N}} G_{k}$ is empty. Indeed if there was some $\boldsymbol{x}$ in $\bigcap_{k \in \mathbb{N}} G_{k}$ then we would have $x+k \boldsymbol{d} \in G$ for every $k \in \mathbb{N}$ which would imply that $x+\uparrow \boldsymbol{d} \sqsubseteq G$ and hence $\boldsymbol{d} \in 0^{+} G$. Observe 
that $G_{1} \cap C_{0}=G \cap C_{0}$ is compact as it is a bounded closed subset of $\mathbb{R}^{n}$. Since $\left(G_{k} \cap C_{0}\right)_{k>1}$ is a non-increasing (w.r.t. inclusion) sequence of closed sets, it follows that $G_{k} \cap C_{0}=\emptyset$ for some $k \geq 1$, and hence $\llbracket a^{k} \rrbracket\left(C_{0}\right)=\emptyset$ for some $k \geq 1$. Moreover, we deduce that $\llbracket a^{*} \rrbracket\left(C_{0}\right)=\bigsqcup_{i \in \mathbb{N}} \llbracket a^{i} \rrbracket\left(C_{0}\right)=\bigsqcup_{i=0}^{k-1} \llbracket a^{i} \rrbracket\left(C_{0}\right)$.

\section{Proof of Proposition 4.4}

We first need the following technical lemma, which can be proved using standard linear algebra techniques.

Lemma C.1. Let $\boldsymbol{x}_{1}, \boldsymbol{x}_{\mathbf{2}}, \boldsymbol{y}_{1}, \boldsymbol{y}_{\mathbf{2}}$ denote four distinct vectors in $\mathbb{R}^{n}$. If we have $\boldsymbol{x}_{\mathbf{2}}-$ $\boldsymbol{x}_{\mathbf{1}}=\lambda\left(\boldsymbol{y}_{\mathbf{2}}-\boldsymbol{y}_{\mathbf{1}}\right)$ for some $\lambda \leq 0$ then there exists $\left.\lambda_{1}, \lambda_{2} \in\right] 0,1\left[\right.$ such that $\lambda_{1} \boldsymbol{x}_{1}+$ $\left(1-\lambda_{1}\right) \boldsymbol{y}_{\mathbf{1}}=\lambda_{2} \boldsymbol{x}_{\mathbf{2}}+\left(1-\lambda_{2}\right) \boldsymbol{y}_{\mathbf{2}}$.

Proposition 4.4. For any $n$-dim action $a=(G, d)$ and for any closed convex set $C_{0} \in$ $\mathrm{C}_{n}$, we have:

$$
\llbracket a \rrbracket^{*}\left(C_{0}\right)= \begin{cases}C_{0} & \text { if } G \cap C_{0}=\emptyset \\ C_{0} \sqcup\left(\left(G \cap\left(C_{0}+\uparrow \boldsymbol{d}\right)\right)+\boldsymbol{d}\right) & \text { otherwise }\end{cases}
$$

Proof. If $G \cap C_{0}=\emptyset$ then $\llbracket a \rrbracket\left(C_{0}\right)=\emptyset$ and therefore $\llbracket a \rrbracket^{*}\left(C_{0}\right)=C_{0}$. If $\boldsymbol{d}=\mathbf{0}$ then $C_{0} \sqcup\left(\left(G \cap\left(C_{0}+\uparrow \boldsymbol{d}\right)\right)+\boldsymbol{d}\right)=C_{0}=\llbracket a \rrbracket^{*}\left(C_{0}\right)$. Now assume for the rest of the proof that $G \cap C_{0} \neq \emptyset$ and $\boldsymbol{d} \neq \mathbf{0}$, and let us write $E=G \cap\left(C_{0}+\uparrow \boldsymbol{d}\right)$. We first prove that $\llbracket a \rrbracket^{*}\left(C_{0}\right) \sqsubseteq\left(C_{0} \sqcup(E+\boldsymbol{d})\right)$. Observe that $\left(C_{0} \sqcup(E+\boldsymbol{d})\right) \sqsubseteq\left(C_{0}+\uparrow \boldsymbol{d}\right)$ since both $C_{0}$ and $E+\boldsymbol{d}$ are closed convex sets that are contained in the closed convex set $\left(C_{0}+\uparrow \boldsymbol{d}\right)$. We therefore get that:

$\llbracket a \rrbracket\left(C_{0} \sqcup(E+\boldsymbol{d})\right)=\left(G \cap\left(C_{0} \sqcup(E+\boldsymbol{d})\right)\right)+\boldsymbol{d} \sqsubseteq\left(G \cap\left(C_{0}+\uparrow \boldsymbol{d}\right)\right)+\boldsymbol{d}=E+\boldsymbol{d}$

Hence we come to $\llbracket a \rrbracket\left(C_{0} \sqcup(E+\boldsymbol{d})\right) \sqsubseteq\left(C_{0} \sqcup(E+\boldsymbol{d})\right)$ and we deduce that $\llbracket a \rrbracket^{*}\left(C_{0}\right) \sqsubseteq$ $\left(C_{0} \sqcup(E+\boldsymbol{d})\right)$. Let us prove the reverse inclusion by contradiction and assume that $\left(C_{0} \sqcup(E+\boldsymbol{d})\right) \nsubseteq \llbracket a \rrbracket^{*}\left(C_{0}\right)$. As $C_{0} \sqsubseteq \llbracket a \rrbracket^{*}\left(C_{0}\right)$ we obtain that there exists $e \in$ $E$ such that $\boldsymbol{e}+\boldsymbol{d} \notin \llbracket a \rrbracket^{*}\left(C_{0}\right)$. Observe that $G \cap \llbracket a \rrbracket^{*}\left(C_{0}\right) \neq \emptyset$. Therefore the set $\left\{\|\boldsymbol{x}-\boldsymbol{e}\|_{\infty} \mid \boldsymbol{x} \in G \cap \llbracket a \rrbracket^{*}\left(C_{0}\right)\right\}$ is non empty and let $\eta$ denote its infimum. Since $G \cap \llbracket a \rrbracket^{*}\left(C_{0}\right)$ is closed, there exists $\boldsymbol{x} \in G \cap \llbracket a \rrbracket^{*}\left(C_{0}\right)$ such that $\|\boldsymbol{x}-\boldsymbol{e}\|_{\infty}=\eta$. Remark that $\boldsymbol{x}^{\prime}=\boldsymbol{x}+\boldsymbol{d} \in \llbracket a \rrbracket^{*}\left(C_{0}\right)$ since $\left(G \cap \llbracket a \rrbracket^{*}\left(C_{0}\right)\right)+\boldsymbol{d}=\llbracket a \rrbracket\left(\llbracket a \rrbracket^{*}\left(C_{0}\right)\right) \sqsubseteq \llbracket a \rrbracket^{*}\left(C_{0}\right)$. As $\boldsymbol{e} \in E$ there exists $\boldsymbol{z} \in C_{0}$ and $\lambda \geq 0$ such that $\boldsymbol{e}=\boldsymbol{z}+\lambda \boldsymbol{d}$. We deduce from Lemma C. 1 applied to $\boldsymbol{z}, \boldsymbol{e}, \boldsymbol{x}^{\prime}, \boldsymbol{x}$ that $\lambda_{1} \boldsymbol{z}+\left(1-\lambda_{1}\right) \boldsymbol{x}^{\prime}=\lambda_{2} \boldsymbol{e}+\left(1-\lambda_{2}\right) \boldsymbol{x}$ for some $\left.\lambda_{1}, \lambda_{2} \in\right] 0,1\left[\right.$. Recall that $\boldsymbol{z}, \boldsymbol{x}^{\prime} \in \llbracket a \rrbracket^{*}\left(C_{0}\right)$ and $\boldsymbol{e}, \boldsymbol{x} \in G$. From convexity of these two sets, we obtain that $\boldsymbol{y}=\left(\lambda_{2} \boldsymbol{e}+\left(1-\lambda_{2}\right) \boldsymbol{x}\right) \in G \cap \llbracket a \rrbracket^{*}\left(C_{0}\right)$. Therefore, we come to $\|\boldsymbol{y}-\boldsymbol{e}\|_{\infty}=\left\|\left(1-\lambda_{2}\right)(\boldsymbol{x}-\boldsymbol{e})\right\|_{\infty}=\left(1-\lambda_{2}\right) \eta<\eta$, a contradiction since $\eta$ is the infimum of $\left\{\|\boldsymbol{x}-\boldsymbol{e}\|_{\infty} \mid \boldsymbol{x} \in G \cap \llbracket a \rrbracket^{*}\left(C_{0}\right)\right\}$.

\section{Proof of lemma 5.2}

We first prove the following lemma. 
Lemma D.1. We have the following equality for any $h \geq 0$ :

$$
\llbracket a_{1} \rrbracket\left(\Lambda_{\mathcal{E}_{2}, h}\left(X_{1}\right)\right)=\operatorname{conv}\left(\left\{(-2,2),(-2,1),\left(-2+\frac{1}{4-h}, 1\right)\right\}\right)
$$

Proof. Let us denote by $C=\llbracket a_{1} \rrbracket\left(\Lambda_{\mathcal{E}_{2}, h}\left(X_{1}\right)\right)$. Following the definitions of $a_{1}$ and $\Lambda_{\mathcal{E}_{2}, h}\left(X_{1}\right)$ we get this equality:

$$
C=(]-\infty,-1] \times[1,+\infty[) \cap \operatorname{conv}(\{(-2,2),(-2,-2),(-1,-2),(-1,-2+h)\})
$$

Let $C^{\prime}=\operatorname{conv}\left(\left\{(-2,2),(-2,1),\left(-2+h^{\prime}, 1\right)\right\}\right)$ where $h^{\prime}=\frac{1}{4-h}$ and let us prove that $C=C^{\prime}$. The following equalities proves that $(-2,-1)$ and $\left(-2+h^{\prime}, 1\right)$ are both in $C$.

$$
\begin{aligned}
& (-2,1)=\frac{1}{4} \cdot(-2,-2)+\frac{3}{4} \cdot(-2,2) \\
& \left(-2+h^{\prime}, 1\right)=h^{\prime} \cdot(-1,-2+h)+\left(1-h^{\prime}\right) \cdot(-2,2)
\end{aligned}
$$

Moreover, from $(-2,2) \in C$, we have proved that $C^{\prime} \subseteq C$. For the other inclusion, let $\boldsymbol{x} \in C$. As $\boldsymbol{x} \in \Lambda_{\mathcal{E}_{2}, h}\left(X_{1}\right)$, there exists $\lambda_{1}, \lambda_{2}, \lambda_{3}, \lambda_{4} \in \mathbb{R}_{+}$such that

$$
\begin{gathered}
\lambda_{1}+\lambda_{2}+\lambda_{3}+\lambda_{4}=1 \\
\boldsymbol{x}=\lambda_{1} \cdot(-2,2)+\lambda_{2} \cdot(-2,-2)+\lambda_{3} \cdot(-1,-2)+\lambda_{4} \cdot(-1,-2+h)
\end{gathered}
$$

Observe that the following equalities hold:

$$
\begin{array}{ll}
(-2,-2) & =4 \cdot(-2,1)-3 \cdot(-2,2) \\
(-1,-2) & =\left(4-\frac{1}{h^{\prime}}\right) \cdot(-2,1)+\frac{1}{h^{\prime}} \cdot\left(-2+h^{\prime}, 1\right)-3 \cdot(-2,2) \\
(-1,-2+h) & =\frac{1}{h^{\prime}} \cdot\left(-2+h^{\prime}, 1\right)-\left(\frac{1}{h^{\prime}}-1\right) \cdot(-2,2)
\end{array}
$$

Thus, by replacing $(-2,-2),(-1,-2),(-1,-2+h)$ by the previous expressions in the linear convex sum decomposing $\boldsymbol{x}$, we get:

$$
\begin{aligned}
\boldsymbol{x} & =\left(\lambda_{1}-3 \cdot \lambda_{2}-3 \cdot \lambda_{3}-\lambda_{4} \cdot\left(\frac{1}{h^{\prime}}-1\right)\right) \cdot(-2,2) \\
& +\left(4 \cdot \lambda_{2}+\lambda_{3} \cdot\left(4-\frac{1}{h^{\prime}}\right)\right) \cdot(-2,1) \\
& +\frac{\lambda_{4}+\lambda_{3}}{h^{\prime}} \cdot\left(-2+h^{\prime}, 1\right)
\end{aligned}
$$

From $x_{2} \geq 1$, the previous equality and $\lambda_{1}+\lambda_{2}+\lambda_{3}+\lambda_{4}=1$, we get:

$$
\left(\lambda_{1}-3 \cdot \lambda_{2}-3 \cdot \lambda_{3}-\lambda_{4} \cdot\left(\frac{1}{h^{\prime}}-1\right)\right) \geq 0
$$

Moreover, as $h \geq 0$ we deduce that $h^{\prime} \geq \frac{1}{4}$ and in particular $4 . \lambda_{2}+\lambda_{3} \cdot\left(4-\frac{1}{h^{\prime}}\right) \geq 0$. We have proved that $\boldsymbol{x} \in C^{\prime}$. Thus $C \subseteq C^{\prime}$.

Lemma 5.2. We have $(\mathbb{1} \sqcup \llbracket T \rrbracket)\left(\Lambda_{\mathcal{E}_{2}, h}\right)=\Lambda_{\mathcal{E}_{2}, \frac{1}{4-h}}$ for any $0 \leq h \leq 2-\sqrt{3}$. 
Proof. From the previous lemma and the definition of $\Lambda_{\varepsilon_{2}, h}$, we deduce the following equality:

$$
\begin{aligned}
& \Lambda_{\mathcal{E}_{2}, h}\left(X_{2}\right) \sqcup \llbracket a_{1} \rrbracket\left(\Lambda_{\mathcal{E}_{2}, h}\left(X_{1}\right)\right) \\
& =\operatorname{conv}\left(\left\{(2,2),(-2,2),(-2,1),(-2+h, 1),\left(-2+\frac{1}{4-h}, 1\right)\right\}\right)
\end{aligned}
$$

Observe that $2-\sqrt{3}$ and $2+\sqrt{3}$ are the two roots of the polynomial $x^{2}-4 . x+1=0$. Thus from $h \leq 2-\sqrt{3}$ we get $h^{2}-4 . h+1 \geq 0$. In particular $-2 \leq-2+h \leq$ $-2+\frac{1}{4-h}$ and we have proved that $\Lambda_{\mathcal{E}_{2}, h}\left(X_{2}\right) \sqcup \llbracket a_{1} \rrbracket\left(\Lambda_{\mathcal{E}_{2}, h}\left(X_{1}\right)\right)=\bar{\Lambda}_{\mathcal{E}_{2}, \frac{1}{4-h}}\left(X_{2}\right)$. By symmetrical rotations, we get the following equalities:

$$
\begin{aligned}
& \left(\mathbb{1} \sqcup \llbracket t_{1} \rrbracket\right)\left(\Lambda_{\mathcal{E}_{2}, d}\right)\left(X_{2}\right)=\Lambda_{\mathcal{E}_{2}, \frac{1}{4-d}}\left(X_{2}\right) \\
& \left(\mathbb{1} \sqcup \llbracket t_{2} \rrbracket\right)\left(\Lambda_{\mathcal{E}_{2}, d}\right)\left(X_{3}\right)=\Lambda_{\mathcal{E}_{2}, \frac{1}{4-d}}\left(X_{3}\right) \\
& \left(\mathbb{1} \sqcup \llbracket t_{3} \rrbracket\right)\left(\Lambda_{\mathcal{E}_{2}, d}\right)\left(X_{4}\right)=\Lambda_{\mathcal{E}_{2}, \frac{1}{4-d}}\left(X_{4}\right) \\
& \left(\mathbb{1} \sqcup \llbracket t_{4} \rrbracket\right)\left(\Lambda_{\mathcal{E}_{2}, d}\right)\left(X_{1}\right)=\Lambda_{\mathcal{E}_{2}, \frac{1}{4-d}}\left(X_{1}\right)
\end{aligned}
$$

Since the variables $X_{1}, X_{2}, X_{3}, X_{4}$ are distinct, we deduce that $(\mathbb{1} \sqcup \llbracket T \rrbracket)\left(\Lambda_{\varepsilon_{2}, d}\right)=$ $\Lambda_{\mathcal{E}_{2}, \frac{1}{4-d}}$.

\section{E Proof of Lemma 5.3}

Recall that $\left(h_{k}\right)_{k \geq 0}$ is the sequence defined by $h_{0}=0$ and $h_{k+1}=\frac{1}{4-h_{k}}$ for any $k \geq 0$.

Lemma 5.3. The sequence $\left(h_{k}\right)_{k \geq 0}$ converges toward $2-\sqrt{3}$.

Proof. Note that $2-\sqrt{3}<2+\sqrt{3}$ are the two roots of the polynomial $x^{2}-4 . x+1=0$. In particular $x^{2}-4 . x+1 \geq 0$ for any $x \leq 2-\sqrt{3}$. Let us first prove by induction that $0 \leq h_{k} \leq 2-\sqrt{3}$. The rank $k=0$ is immediate since $d_{0}=0$. Observe that $0 \leq h_{k} \leq 2-\sqrt{3}$ implies $\frac{1}{4} \leq h_{k+1} \leq \frac{1}{4-(2-\sqrt{3})}=2-\sqrt{3}$. We have proved that $0 \leq h_{k} \leq 2-\sqrt{3}$ for any $k \geq 0$. From $h_{k+1}-h_{k}=\frac{h_{k}^{2}-4 . h_{k}+1}{4-h_{k}}$ and $0 \leq h_{k}<2-\sqrt{3}$ we get $h_{k+1}-h_{k}>0$. Thus $\left(h_{k}\right)_{k \geq 0}$ is a bounded increasing sequence, We deduce that $\left(h_{k}\right)_{k \geq 0}$ converges toward a limit $h$. Taking the limit in the equality $h_{k+1} \cdot\left(4-h_{k}\right)=1$ and the inequality $h_{k} \leq 2-\sqrt{3}$ provides $h .(4-h)=1$ and $h \leq 2-\sqrt{3}$. Thus $h=2-\sqrt{3}$.

\section{F Proof of Lemma 5.5}

Recall that the sequence $\left(z_{i}\right)_{i \geq 1}$ is defined by $z_{0}=\frac{3}{2}$ and $z_{i+1}=1+z_{i} \cdot \frac{i}{i+1}$ for any $i \geq 1$, and the sequence $\left(h_{i}\right)_{i \geq 1}$ is defined by $h_{i}=\frac{1}{i}$ for any $i \geq 1$.

Lemma F.1. The sequence $\left(z_{i}\right)_{i \geq 1}$ is unbounded, increasing and it satisfies $z_{i}<i+1$ for any $i \geq 1$. 
Proof. An immediate induction provides $z_{i}<i+1$ for any $i \geq 1$. From $z_{i+1}=$ $1+z_{i} \cdot \frac{i}{i+1}$, we get $z_{i+1}-z_{i}=\frac{(i+1)-z_{i}}{i+2}$ thus $z_{i+1}>z_{i}$ and we have proved that $z_{i}$ is an increasing sequence. Note that if $\left(z_{i}\right)_{i \geq 1}$ is bounded, we deduce that $z_{i}$ converges toward a vector $z$. Taking the limit in the equality $z_{i+1}=1+z_{i} \cdot \frac{i}{i+1}$ provides $z=1+z$ and we get a contradiction. Therefore $\left(z_{i}\right)_{i \geq 1}$ is unbounded.

A function $f \in C \rightarrow \mathbb{R}$ is said convex if its graph $G_{f}=\{(\boldsymbol{x}, y) \in C \times \mathbb{R} \mid y \geq$ $f(\boldsymbol{x})\}$ is convex. Let us prove that function $f$ defined over ] 0,1$]$ by the following equality for any $\left.\lambda \in] h_{i+1}, h_{i}\right]$ and for any $i \geq 1$ :

$$
f(\lambda)=z_{i+1} \cdot \frac{\lambda-h_{i}}{h_{i+1}-h_{i}}+z_{i} \cdot \frac{\lambda-h_{i+1}}{h_{i}-h_{i+1}}
$$

Lemma F.2. Function $f$ is convex.

Proof. Observe that it is sufficient to show the following inequality for any $i \geq 2$ :

$$
-\frac{f\left(h_{i+1}\right)-f\left(h_{i}\right)}{h_{i+1}-h_{i}}+\frac{f\left(h_{i}\right)-f\left(h_{i-1}\right)}{h_{i}-h_{i-1}}>0
$$

By replacing $h_{i-1}, h_{i}, h_{i+1}, f\left(h_{i-1}\right), f\left(h_{i}\right), f\left(h_{i+1}\right)$ respectively by $\frac{1}{i-1}, \frac{1}{i}, \frac{i}{i-1} \cdot\left(z_{i}-\right.$ 1), $z_{i}, 1+z_{i} \cdot \frac{i}{i+1}, z_{i}$, the previous difference becomes equal to $i+z_{i}(i-1)>0$.

We now prove that values of $\Lambda_{\mathcal{S}_{3},+\infty}$ in $X_{1}, X_{2}, X_{3}, X_{4}$ are closed (this result is not immediate).

Lemma F.3. The set $\operatorname{conv}\left(\left\{\left(h_{i}, z_{i}\right) \mid i \geq 1\right\}\right)+\uparrow(0,1)$ is closed.

Proof. Let $C$ be this closed convex set. Observe that $C$ is the graph of the function $f$. Consider a sequence $\left(\left(x_{j}, y_{j}\right)\right)_{j \geq 0}$ in this graph that converges toward a vector $(\lambda, y)$. Note that there exists $i_{j} \in \mathbb{N} \backslash\{0\}$ such that $\left.\left.x_{j} \in\right] h_{i_{j}+1}, h_{i_{j}}\right]$. Since $y_{j} \geq f\left(x_{j}\right) \geq z_{i_{j}}$ we deduce that $\left(z_{i_{j}}\right)_{j \geq 0}$ is bounded. As $\left(z_{i}\right)_{i \geq 1}$ is increasing and unbounded, we deduce that $\left(i_{j}\right)_{j \geq 0}$ is bounded. Thus, by extracting subsequences, we can assume that $\left(i_{j}\right)_{j \geq 0}$ remains equal to a constant $i \in \mathbb{N} \backslash\{0\}$. Thus $\left(x_{j}\right)_{j \geq 0}$ converges toward $x \in\left[h_{i+1}, h_{i}\right]$. Since $f$ is continuous over $\left[h_{i+1}, h_{i}\right]$, from $y_{j} \geq f\left(x_{j}\right)$ we deduce $y \geq f(x)$. Thus $(x, y)$ is in the graph of $f$ and we have proved that $C$ is closed.

Lemma 5.5. Values of $\Lambda_{\varepsilon_{3},+\infty}$ in $X_{1}, X_{2}, X_{3}, X_{4}$ are closed convex sets but they are not polyhedral.

Proof. Let $C$ be the convex hull of $\left\{\left(0,-h_{i}, z_{i}\right) \mid i \geq 1\right\}$. From the previous lemma F.3 we deduce that $C+\uparrow e_{3}$ is closed. Note that there exists a bounded closed convex set $C_{0}$ such that $C+\uparrow d=C_{0}+\uparrow d$. Since $C_{0}$ is a bounded closed convex set, we deduce that the convex hull of $(-1,1,0) \cup(-1,-1,1) \cup C_{0}$ is a closed convex set $C^{\prime}$. Now, just observe that $\Lambda_{\mathcal{S}_{3},+\infty}\left(X_{1}\right)=C^{\prime}+\uparrow \boldsymbol{e}_{3}$. Thus this set is closed. By symmetry, we have proved that values of $\Lambda_{\mathfrak{S}_{3},+\infty}$ in $X_{1}, X_{2}, X_{3}, X_{4}$ are closed.

Now, let us prove that these values are not polyhedral. Observe that if $\Lambda_{\mathcal{E}_{3},+\infty}\left(X_{1}\right)$ is polyhedral then $\Lambda_{\varepsilon_{3},+\infty}\left(X_{1}\right) \cap\{0\} \times \mathbb{R}^{2}$ is also polyhedral. Note that this set is equal to $C+e_{3}$. Since the graph of $f$ is not polyhedral we deduce that $\Lambda_{\varepsilon_{3},+\infty}\left(X_{1}\right)$ is not polyhedral. By symmetry, the value of $\Lambda_{\varepsilon_{3},+\infty}$ in $X_{1}, X_{2}, X_{3}, X_{4}$ are not polyhedral. 


\section{G Proof of Lemma 5.6}

Lemma G.1. The set $\llbracket a_{1} \rrbracket\left(\Lambda_{\varepsilon_{3}, k}\left(X_{1}\right)\right)$ is equal to the following set for any $k \in\{2, \ldots,+\infty\}$ :

$$
\operatorname{conv}\left(\{(-1,1,1)\} \cup\left\{\left(-h_{i+1}, 0, z_{i+1}\right) \mid 0 \leq i<k\right\}\right)+\uparrow \boldsymbol{e}_{\mathbf{3}}
$$

Proof. Let us denote by $C=\llbracket a_{1} \rrbracket\left(\Lambda_{\varepsilon_{3}, k}\left(X_{1}\right)\right)$. Following definitions of $a_{1}$ and $\Lambda_{\varepsilon_{3}, k}\left(X_{1}\right)$, we get this equality:

$C-\boldsymbol{e}_{\mathbf{3}}=\left(\mathbb{R}_{-} \times \mathbb{R}_{+} \times \mathbb{R}\right) \cap\left(\operatorname{conv}\left(\{(-1,1,0),(-1,-1,1)\} \cup\left\{\left(0,-h_{i}, z_{i}\right) \mid 1 \leq i<k\right\}\right)+\uparrow \boldsymbol{e}_{\mathbf{3}}\right)$

Note that the following equalities hold:

$$
\begin{array}{ll}
\left(-h_{i+1}, 0, z_{i+1}\right)-e_{\mathbf{3}} & =\frac{1}{i+1} \cdot(-1,1,0)+\frac{i}{i+1} \cdot\left(0,-h_{i}, z_{i}\right) \\
\left(-h_{1}, 0, z_{1}\right)-e_{3} & =\frac{1}{2} \cdot(-1,-1,1)+\frac{1}{2} \cdot(-1,1,0) \\
(-1,1,1)-e_{3} & =(-1,1,0)
\end{array}
$$

Thus $\left(-h_{i+1}, 0, z_{i+1}\right),\left(-h_{1}, 0, z_{1}\right)$ and $(-1,1,1)$ are in $C$ and we have proved that $C$ contains the following convex set $C^{\prime}$ :

$$
C^{\prime}=\operatorname{conv}\left(\left\{(-1,1,1) \cup\left\{\left(-h_{i+1}, 0, z_{i+1}\right) \mid 0 \leq i \leq k\right\}\right)+\uparrow e_{3}\right.
$$

For the converse inclusion, let $\boldsymbol{x} \in C$. There exists $\lambda, \mu, \beta \in \mathbb{R}_{+}$and a sequence $\left(r_{i}\right)_{1 \leq i<k}$ of elements in $\mathbb{R}_{+}$such that $r_{i}=0$ expect for a finite number of $i$ such that:

$$
\begin{gathered}
\lambda+\mu+\sum_{1 \leq i<k} r_{i}=1 \\
\boldsymbol{x}-\boldsymbol{e}_{\mathbf{3}}=\lambda \cdot(-1,1,0)+\mu \cdot(-1,-1,1)+\sum_{1 \leq i<k} r_{i} \cdot\left(0,-h_{i}, z_{i}\right)+\beta . \boldsymbol{e}_{\mathbf{3}}
\end{gathered}
$$

Observe that the two following equalities hold:

$$
\begin{aligned}
& \left(0,-h_{i}, z_{i}\right)=\frac{i+1}{i} \cdot\left(-h_{i+1}, 0, z_{i+1}-1\right)-\frac{1}{i+1} \cdot(-1,1,0) \\
& (-1,-1,1)=2 \cdot\left(-h_{1}, 0, z_{1}-1\right)-(-1,1,0)
\end{aligned}
$$

Thus, by replacing $\left(0,-h_{i}, z_{i}\right)$ and $(-1,-1,1)$ by the previous expressions in the linear convex sum decomposing $\boldsymbol{x}-\boldsymbol{e}_{3}$, we get:

$$
\begin{aligned}
\boldsymbol{x}-\boldsymbol{e}_{\mathbf{3}}= & \left(\lambda-\mu-\sum_{1 \leq i<k} \frac{r_{i}}{i+1}\right) \cdot(-1,1,0) \\
& +2 \cdot \mu \cdot\left(-h_{1}, 0, z_{1}-1\right) \\
& +\sum_{1 \leq i<k} \frac{i+1}{i} \cdot r_{i} \cdot\left(-h_{i+1}, 0, z_{i+1}-1\right) \\
& +\beta \cdot \boldsymbol{e}_{\mathbf{3}}
\end{aligned}
$$

From the following equality:

$$
\boldsymbol{e}_{\mathbf{3}}=\left(\lambda-\mu-\sum_{1 \leq i<k} \frac{r_{i}}{i+1}\right) \cdot \boldsymbol{e}_{\mathbf{3}}+2 \cdot \mu \cdot \boldsymbol{e}_{\mathbf{3}}+\sum_{1 \leq i<k} \frac{i+1}{i} \cdot r_{i} \cdot \boldsymbol{e}_{\mathbf{3}}
$$


We get:

$$
\begin{aligned}
\boldsymbol{x}= & \left(\lambda-\mu-\sum_{1 \leq i<k} \frac{r_{i}}{i+1}\right) \cdot(-1,1,1) \\
& +2 . \mu \cdot\left(-h_{1}, 0, z_{1}\right) \\
& +\sum_{1 \leq i<k} \frac{i+1}{i} \cdot r_{i} \cdot\left(-h_{i+1}, 0, z_{i+1}\right) \\
& +\beta \cdot \boldsymbol{e}_{3}
\end{aligned}
$$

Since $\boldsymbol{x}-\boldsymbol{e}_{\mathbf{3}} \in \mathbb{R}_{-} \times \mathbb{R}_{+} \times \mathbb{R}$, we deduce that $x_{2} \geq 0$. Thus $\left(\lambda-\mu-\sum_{1 \leq i<k} \frac{r_{i}}{i+1}\right) \geq 0$. As $\left(\lambda-\mu-\sum_{1 \leq i<k} \frac{r_{i}}{i+1}\right)+2 . \mu+\sum_{1 \leq i<k} \frac{i+1}{i} . r_{i}=1$ we deduce $\boldsymbol{x} \in C^{\prime}$.

Lemma 5.6. We have $(\mathbb{1} \sqcup \llbracket T \rrbracket)\left(\Lambda_{\varepsilon_{3}, k}\right)=\Lambda_{\varepsilon_{3}, k+1}$ for any $k \in\{2, \ldots,+\infty\}$.

Proof. From lemma G.1, we deduce that $\Lambda_{\varepsilon_{3}, k}\left(X_{2}\right) \sqcup \llbracket a_{1} \rrbracket\left(\Lambda_{\varepsilon_{3}, k}\left(X_{1}\right)\right)$ is equal to $\Lambda_{\mathcal{E}_{3}, k+1}\left(X_{2}\right)$. Thus $\left(\mathbb{1} \sqcup \llbracket t_{1} \rrbracket\right)\left(\Lambda_{\mathcal{E}_{3}, k}\right)\left(X_{2}\right)=\Lambda_{\mathcal{E}_{3}, k+1}\left(X_{2}\right)$. By symmetrical rotations, we get the following equalities:

$$
\begin{aligned}
& \left(\mathbb{1} \sqcup \llbracket t_{1} \rrbracket\right)\left(\Lambda_{\varepsilon_{3}, k}\right)\left(X_{2}\right)=\Lambda_{\varepsilon_{3}, k+1}\left(X_{2}\right) \\
& \left(\mathbb{1} \sqcup \llbracket t_{2} \rrbracket\right)\left(\Lambda_{\mathcal{E}_{3}, k}\right)\left(X_{3}\right)=\Lambda_{\mathcal{E}_{3}, k+1}\left(X_{3}\right) \\
& \left(\mathbb{1} \sqcup \llbracket t_{3} \rrbracket\right)\left(\Lambda_{\varepsilon_{3}, k}\right)\left(X_{4}\right)=\Lambda_{\varepsilon_{3}, k+1}\left(X_{4}\right) \\
& \left(\mathbb{1} \sqcup \llbracket t_{4} \rrbracket\right)\left(\Lambda_{\varepsilon_{3}, k}\right)\left(X_{1}\right)=\Lambda_{\varepsilon_{3}, k+1}\left(X_{1}\right)
\end{aligned}
$$

Since the variables $X_{1}, X_{2}, X_{3}, X_{4}$ are distinct, we deduce $(\mathbb{1} \sqcup \llbracket T \rrbracket)\left(\Lambda_{\varepsilon_{3}, k}\right)=$ $\Lambda_{\varepsilon_{3}, k+1}$.

\section{H Proof of Lemma 6.2}

Lemma 6.2. We have cloconv $((G \cap S)+\boldsymbol{d})=(G \cap \operatorname{cloconv}(S))+\boldsymbol{d}$ for any $n$-dim action $a=(G, \boldsymbol{d})$ and for any set $S \subseteq \mathbb{R}^{n}$ such that bd $(G) \cap \operatorname{cloconv}(S) \subseteq S$.

Proof. Naturally, we can assume that $\boldsymbol{d}=\mathbf{0}$. Let us prove the non immediate inclusion $G \cap \operatorname{cloconv}(S) \sqsubseteq \operatorname{cloconv}(G \cap S)$. Let $\boldsymbol{x} \in G \cap \operatorname{cloconv}(S)$. Observe that if $\boldsymbol{x} \in \operatorname{bd}(G)$ then from $\boldsymbol{x} \in \operatorname{bd}(G) \cap \operatorname{cloconv}(S) \subseteq S$, we get $\boldsymbol{x} \in G \cap S$ and in particular $\boldsymbol{x} \in \operatorname{cloconv}(G \cap S)$. Thus, we can assume that $\boldsymbol{x} \in G \backslash \operatorname{bd}(G)$. Since $\boldsymbol{x} \in \operatorname{cloconv}(S)$, there exits a sequence $\left(S_{k}\right)_{k \geq 0}$ of finite subsets of $S$ and a sequence $\left(\boldsymbol{x}_{\boldsymbol{k}}\right)_{k \geq 0}$ in the convex hull of $S_{k}$ that converges toward $\boldsymbol{x}$. As $\boldsymbol{x}$ is in the interior $G \backslash \operatorname{bd}(G)$ of $G$, there exists an integer $K \geq 0$ such that $\boldsymbol{x}_{\boldsymbol{k}}$ is also in this set for any $k \geq K$. By re-indexing the sequence, we can assume that $K=0$. Let us consider a sequence $\left(\lambda_{k, \boldsymbol{y}}\right)_{\boldsymbol{y} \in S_{k}}$ in $\mathbb{R}_{+}$such that $\sum_{\boldsymbol{y} \in S_{k}} \lambda_{k, \boldsymbol{y}}=1$ and such that $\boldsymbol{x}_{\boldsymbol{k}}$ is a linear convex combination $\boldsymbol{x}_{\boldsymbol{k}}=\sum_{\boldsymbol{y} \in S_{k}} \lambda_{k, \boldsymbol{y}} \cdot \boldsymbol{y}$. Observe that for any $\boldsymbol{y} \in S_{k} \backslash G$, as $\boldsymbol{x}_{\boldsymbol{k}} \in G \backslash \mathrm{bd}(G)$, there exist a real value $\mu_{k, \boldsymbol{y}}$ such that $0<\mu_{k, \boldsymbol{y}}<1$ and such that $\left(1-\mu_{k, \boldsymbol{y}}\right) \cdot \boldsymbol{x}_{\boldsymbol{k}}+\mu_{k, \boldsymbol{y}} \cdot \boldsymbol{y} \in \operatorname{bd}(G)$. Let us denote by $f_{k}(\boldsymbol{y})$ this vector in bd $(G)$. Since $\boldsymbol{x}_{\boldsymbol{k}}$ is a convex linear combination of vectors in $S$ and $\boldsymbol{y} \in S$ we deduce that $f_{k}(\boldsymbol{y})$ is also a convex linear combination of vectors in $S$. Thus $f_{k}(\boldsymbol{y}) \in$ 
bd $(G) \cap \operatorname{conv}(S) \subseteq S$ and we have proved that $f_{k}(\boldsymbol{y}) \in G \cap S$. By replacing each $\boldsymbol{y} \in S_{k} \backslash G$ by $\frac{1}{\mu_{k, \boldsymbol{y}}} \cdot\left(f_{k}(\boldsymbol{y})-\left(1-\mu_{k, \boldsymbol{y}}\right) \cdot \boldsymbol{x}_{\boldsymbol{k}}\right)$ in the equality $\boldsymbol{x}_{\boldsymbol{k}}=\sum_{\boldsymbol{y} \in S_{k}} \lambda_{k, \boldsymbol{y}} \cdot \boldsymbol{y}$, we get:

$$
\left(\sum_{\boldsymbol{y} \in S_{k} \cap G} \lambda_{k, \boldsymbol{y}}+\sum_{\boldsymbol{y} \in S_{k} \backslash G} \frac{\lambda_{k, \boldsymbol{y}}}{\mu_{k, \boldsymbol{y}}}\right) \cdot x_{k}=\sum_{\boldsymbol{y} \in S_{k} \cap G} \lambda_{k, \boldsymbol{y}} \cdot \boldsymbol{y}+\sum_{\boldsymbol{y} \in S_{k} \backslash G} \frac{\lambda_{k, \boldsymbol{y}}}{\mu_{k, \boldsymbol{y}}} \cdot f_{k}(\boldsymbol{y})
$$

Therefore $\boldsymbol{x}_{\boldsymbol{k}}$ is a linear convex combination of vectors in $G \cap S$. Since $\boldsymbol{x}_{\boldsymbol{k}}$ converges toward $\boldsymbol{x}$, we deduce that $\boldsymbol{x} \in \operatorname{cloconv}(G \cap S)$.

\section{Proof of Lemma 6.3}

Lemma 6.3. We have the following equality :

$$
\Lambda_{\mathcal{S}}(X)=\bigsqcup\left\{\llbracket \sigma \rrbracket\left(\Delta_{\mathcal{S}}\left(X_{0}\right)\right) \mid X_{0} \in \mathcal{X}, \sigma \in L_{X_{0}, X}^{E}\right\}+0^{+} \Lambda_{\mathcal{S}}(X)
$$

Proof. Let $\Lambda_{\mathrm{S}}^{\prime \prime}$ be the valuation defined by the following equality:

$$
\Lambda_{\mathcal{S}}^{\prime \prime}(X)=\bigsqcup\left\{\llbracket \sigma \rrbracket\left(\Delta_{\mathcal{S}}\left(X_{0}\right)\right) \mid X_{0} \in \mathcal{X}, \sigma \in L_{X_{0}, X}^{E}\right\}+0^{+} \Lambda_{\mathcal{S}}(X)
$$

As $L_{X_{0}, X}^{E} \subseteq L_{X_{0}, X}$ we deduce that $\bigsqcup\left\{\llbracket \sigma \rrbracket\left(\Delta_{\mathcal{S}}\left(X_{0}\right)\right) \mid X_{0} \in \mathcal{X}, \sigma \in L_{X_{0}, X}^{E}\right\} \sqsubseteq$ $\Lambda_{\mathcal{S}}^{\prime}(X)$. Moreover as $\Lambda_{\mathcal{S}}(X)+0^{+} \Lambda_{\mathcal{S}}(X)=\Lambda_{\mathcal{S}}(X)$ and $\Lambda_{\mathcal{S}}=\Lambda_{\mathcal{S}}^{\prime}$ we get $\Lambda_{\mathcal{S}}^{\prime \prime}(X) \sqsubseteq$ $\Lambda_{\mathcal{S}}^{\prime}(X)$.

Now, assume by contradiction that the inclusion is strict. We deduce that there exists a path $\pi=\left(X_{0} \stackrel{\sigma}{\rightarrow} X\right)$ and a vector $\boldsymbol{x}_{\mathbf{0}} \in \Delta_{\mathcal{S}}\left(X_{0}\right)$ such that $\llbracket \sigma \rrbracket\left(\boldsymbol{x}_{\mathbf{0}}\right)$ is reduced to a vector denoted by $\boldsymbol{x}$ satisfying $\boldsymbol{x} \in \Lambda_{\mathrm{S}}^{\prime}(X)$ and such that $\boldsymbol{x} \notin \Lambda_{\mathrm{S}}^{\prime \prime}(X)$. Naturally, we can assume without loss of generality that the length of $\pi$ is minimal.

Note that $\pi$ cannot be simple. Thus, this path can be decomposed into $\pi=\pi_{0} \cdot \theta \cdot \pi_{1}$ where $\pi_{0}=\left(X_{0} \stackrel{\sigma_{0}}{\longrightarrow} X_{1}\right), \theta=\left(X_{1} \stackrel{w}{\longrightarrow} X_{1}\right)$ is a loop with a non zero length and $\pi_{1}=\left(X_{1} \stackrel{\sigma_{1}}{\longrightarrow} X\right)$. We denote by $\boldsymbol{x}_{\mathbf{1}}$ and $\boldsymbol{x}^{\prime}$ the vectors $\boldsymbol{x}_{\boldsymbol{1}}=\boldsymbol{x}_{\mathbf{0}}+\boldsymbol{d}_{\sigma_{0}}, \boldsymbol{x}^{\prime}=$ $\boldsymbol{x}_{\mathbf{1}}+\boldsymbol{d}_{\sigma_{1}}$. Note that $\boldsymbol{x}=\boldsymbol{x}^{\prime}+\boldsymbol{d}_{\boldsymbol{w}}$. When the set $\llbracket \sigma_{0} \cdot w^{k} \cdot \sigma_{1} \rrbracket\left(\boldsymbol{x}_{\mathbf{0}}\right)$ is not empty, it is reduced to $\llbracket \sigma_{1} \rrbracket\left(\boldsymbol{x}_{1}+k \cdot \boldsymbol{d}_{\boldsymbol{w}}\right)$. We denote by $I$ the set of real $r \in \mathbb{R}_{+}$such that $\llbracket \sigma_{1} \rrbracket\left(\boldsymbol{x}_{\boldsymbol{1}}+r . \boldsymbol{d}_{\boldsymbol{w}}\right) \neq \emptyset$. Observe that $I$ is a non empty closed interval of the form $I=\left\{r \in \mathbb{R}_{+} \mid r_{0} \leq r \leq r_{1}\right\}$ where $r_{0} \in \mathbb{R}_{+}$and $r_{1} \in \mathbb{R}_{+} \cup\{+\infty\}$.

Let us show that $\boldsymbol{x}^{\prime}+r_{0} . \boldsymbol{d}_{\boldsymbol{w}} \in \Lambda_{\mathrm{S}}^{\prime \prime}(X)$. Note that if $r_{0}=0$, the path $\pi_{0} \cdot \pi_{1}$ with a smaller length than $\pi$ and the vector $\boldsymbol{x}_{\mathbf{0}} \in \Delta_{\mathcal{\delta}}\left(X_{0}\right)$ provides $\boldsymbol{x}^{\prime}+0 . \boldsymbol{d}_{\boldsymbol{w}} \in \Lambda_{\mathcal{\delta}}^{\prime \prime}(X)$. Now, consider the case $r_{0}>0$. The sequence $t_{1}, \ldots, t_{k}$ of $k \geq 0$ transitions $t_{i}=\left(Y_{i-1} \stackrel{a_{i}}{\longrightarrow}\right.$ $Y_{i}$ ) such that $\pi_{1}=t_{1} \ldots t_{k}$ will be useful for proving the property in this case. In fact, as $r_{0}$ is the minimal real in $\mathbb{R}$ (not only in $\mathbb{R}_{+}$) such that $\llbracket a_{1} \ldots a_{k} \rrbracket\left(\boldsymbol{x}_{1}+r_{0} . \boldsymbol{d}_{\boldsymbol{w}}\right) \neq \emptyset$. We deduce that there exists $1 \leq i \leq k$ such that $\llbracket a_{1} \ldots a_{i-1} \rrbracket\left(\boldsymbol{x}_{\boldsymbol{1}}+r_{0} . \boldsymbol{d}_{\boldsymbol{w}}\right) \in \operatorname{bd}\left(G_{t_{i}}\right)$. Note that $\boldsymbol{x}_{1}$ and $\boldsymbol{x}_{1}+\boldsymbol{d}_{\boldsymbol{w}}$ are both in $\Lambda_{\mathcal{S}}\left(X_{1}\right)$ thanks to the paths $\pi_{0}$ and $\pi_{0} \cdot \theta$ and the vector $\boldsymbol{x}_{\mathbf{0}} \in \Delta_{\mathrm{s}}\left(X_{0}\right)$. As $0 \leq r_{0} \leq 1$ and $\Lambda_{\mathcal{S}}\left(X_{1}\right)$ is convex, we deduce that $\boldsymbol{x}_{\mathbf{1}}+$ $r_{0} . \boldsymbol{d}_{\boldsymbol{w}} \in \Lambda_{\mathcal{S}}\left(X_{1}\right)$. Therefore $\llbracket a_{1} \ldots a_{i-1} \rrbracket\left(\boldsymbol{x}_{\mathbf{1}}+r_{0} . \boldsymbol{d}_{\boldsymbol{w}}\right) \in \Lambda_{\mathcal{S}}\left(Y_{i-1}\right)$ thanks to the path 
$t_{1} \ldots t_{i-1}$ and the vector $\boldsymbol{x}_{\mathbf{1}}+r_{0} \cdot \boldsymbol{d}_{\boldsymbol{w}} \in \Lambda_{\delta}\left(X_{1}\right)$. Thus $\llbracket a_{1} \ldots a_{i-1} \rrbracket\left(\boldsymbol{x}_{\mathbf{1}}+r_{0} \cdot \boldsymbol{d}_{\boldsymbol{w}}\right) \in$ bd $\left(G_{t_{i}}\right) \cap \Lambda_{\mathcal{S}}\left(Y_{i-1}\right) \sqsubseteq \Delta_{\mathcal{S}}\left(Y_{i-1}\right)$. The path $t_{i+1} \ldots t_{k}$ with a smaller length that $\pi$ and the vector $\llbracket a_{1} \ldots a_{i-1} \rrbracket\left(\boldsymbol{x}_{\boldsymbol{1}}+r_{0} . \boldsymbol{d}_{\boldsymbol{w}}\right) \in \Delta_{\mathcal{S}}\left(Y_{i-1}\right)$ prove that $\llbracket a_{1} \ldots a_{k} \rrbracket\left(\boldsymbol{x}_{\boldsymbol{1}}+\right.$ $\left.r_{0} . \boldsymbol{d}_{\boldsymbol{w}}\right) \in \Lambda_{\mathcal{S}}^{\prime \prime}(X)$. Thus $\boldsymbol{x}^{\prime}+r_{0} . \boldsymbol{d}_{\boldsymbol{w}} \in \Lambda_{\mathcal{S}}^{\prime \prime}(X)$.

Finally, note that if $r_{1}=+\infty$ then $\boldsymbol{d}_{\boldsymbol{w}} \in 0^{+} \Lambda_{\mathcal{S}}(X)$. From $\boldsymbol{x}^{\prime}+r_{0} . \boldsymbol{d}_{\boldsymbol{w}} \in \Lambda_{\mathcal{\delta}}^{\prime \prime}(X)$ we deduce that $\boldsymbol{x}=\boldsymbol{x}^{\prime}+r_{0} . \boldsymbol{d}_{\boldsymbol{w}}+\left(1-r_{0}\right) \cdot \boldsymbol{d}_{\boldsymbol{w}} \in \Lambda_{\mathcal{\delta}}^{\prime \prime}(X)$ and we get a contradiction. Thus $r_{1}<+\infty$. A symmetrical proof as the one given in the previous paragraph shows that $\boldsymbol{x}^{\prime}+r_{1} \cdot \boldsymbol{d}_{\boldsymbol{w}} \in \Lambda_{\mathcal{S}}^{\prime \prime}(X)$. As $r_{0} \leq 1 \leq r_{1}$ and $\boldsymbol{x}^{\prime}+r_{0} \cdot \boldsymbol{d}_{\boldsymbol{w}}$ and $\boldsymbol{x}^{\prime}+r_{1} . \boldsymbol{d}_{\boldsymbol{w}}$ are both in the convex set $\Lambda_{\mathcal{S}}^{\prime \prime}(X)$, we deduce that $\boldsymbol{x}=\boldsymbol{x}^{\prime}+\boldsymbol{d}_{\boldsymbol{w}} \in \Lambda_{\mathcal{S}}^{\prime \prime}(X)$ and we also get a contradiction.

\section{J Proof of Theorem 6.5}

Theorem 6.5. The MFP-solution of any 2-dim A-polyhedral IGTS is effectively A-polyhedral.

Proof. We denote by $H_{\alpha, c}$ the half-space $\left\{\boldsymbol{x} \in \mathbb{R}^{2} \mid\langle\boldsymbol{\alpha}, \boldsymbol{x}\rangle \leq c\right\}$ parameterized by $\boldsymbol{\alpha} \in \mathbb{R}^{2}$ and $c \in \mathbb{R}$. Given a tuple $r_{1}=\left(\alpha_{1}, c_{1}, \alpha_{2}, c_{2}\right)$ where $\alpha_{1}=\left(\boldsymbol{\alpha}_{1, t}\right)_{t \in T}$ and $\alpha_{2}=\left(\boldsymbol{\alpha}_{\mathbf{2}, t}\right)_{t \in T}$ are two sequences of elements in $\mathbb{R}^{2}$ and where $c_{1}=\left(c_{1, t}\right)_{t \in T}$ and $c_{2}=\left(c_{2, t}\right)_{t \in T}$ are two sequence of elements in $\mathbb{R}$, we denote by $\Delta_{\mathcal{S}, r_{1}}$ the following valuation:

$$
\Delta_{\delta, r_{1}}(X)=\rho_{0}(X) \sqcup \bigsqcup\left\{\operatorname{bd}(G) \cap H_{\boldsymbol{\alpha}_{1, t}, c_{1, t}} \cap H_{\boldsymbol{\alpha}_{\mathbf{2}, t}, c_{2, t}} \mid t=\left(X \stackrel{a=(G, \boldsymbol{d})}{\longrightarrow} X^{\prime}\right)\right\}
$$

Given a tuple $r_{2}=\left(d_{1}, d_{2}, d_{3}\right)$ where $d_{1}=\left(\boldsymbol{d}_{1, X}\right)_{X \in \mathcal{X}}, d_{2}=\left(\boldsymbol{d}_{2, \boldsymbol{X}}\right)_{X \in \mathcal{X}}$ and $d_{3}=$ $\left(\boldsymbol{d}_{\boldsymbol{3}, \boldsymbol{X}}\right)_{X \in \mathcal{X}}$ are three sequences of elements in $\mathbb{R}^{2}$, we denote by $C_{\mathcal{S}, r_{2}}$ the following valuation:

$$
C_{\mathcal{S}, r_{2}}(X)=\uparrow \boldsymbol{d}_{1, X}+\uparrow \boldsymbol{d}_{2, \boldsymbol{X}}+\uparrow \boldsymbol{d}_{\mathbf{3}, \boldsymbol{X}}
$$

Observe that lemma 6.3 proves that there exists $r=\left(r_{1}, r_{2}\right)$ such that $\Lambda_{\S, r}=\Lambda_{\mathcal{S}}$ where $\Lambda_{\S, r}$ is the following valuation:

$$
\Lambda_{\mathcal{S}, r}(X)=\bigsqcup\left\{\llbracket \sigma \rrbracket\left(\Delta_{\mathcal{S}, r_{1}}\left(X_{0}\right)\right) \mid X_{0} \in \mathcal{X}, \sigma \in L_{X_{0}, X}^{E}\right\}+C_{\S, r_{2}}(X)
$$

Finally, let us consider the following formula $\phi(r)$ :

$$
\phi(r):=\llbracket T \rrbracket\left(\Lambda_{\mathcal{S}, r}\right) \sqsubseteq \Lambda_{\mathcal{S}, r} \wedge \forall r^{\prime}\left(\llbracket T \rrbracket\left(\Lambda_{\mathcal{S}, r^{\prime}}\right) \sqsubseteq \Lambda_{\mathcal{S}, r^{\prime}} \Longrightarrow \Lambda_{\mathcal{S}, r} \sqsubseteq \Lambda_{\mathcal{S}, r^{\prime}}\right)
$$

As the boundary of any guard is definable in $\langle\mathbb{R},+, \cdot\rangle$, we deduce that $\phi$ is a formula in this logic. Note that an element $r^{\prime}$ satisfying $\llbracket T \rrbracket\left(\Lambda_{\mathcal{S}, r^{\prime}}\right) \sqsubseteq \Lambda_{\mathcal{S}, r^{\prime}}$ is a post-fix-point. Moreover, as $\rho_{0} \sqsubseteq \Lambda_{\mathcal{S}, r^{\prime}}$ we deduce that $\Lambda_{\mathcal{S}} \sqsubseteq \Lambda_{\mathcal{S}, r^{\prime}}$. As there exists an $r$ such that $\Lambda_{\delta, r}=\Lambda_{\S}$ we deduce that $\phi$ defines the set of $r$ such that $\Lambda_{\delta, r}=\Lambda_{\S}$. In particular $\phi$ is satisfiable and there exists an effectively computable algebraic solution $r$. Now just observe that $\Lambda_{\mathcal{S}, r}$ is A-polyhedral. 